\title{
RE-GAUGING GROUPOID, SYMMETRIES AND DEGENERACIES FOR GRAPH HAMILTONIANS AND APPLICATIONS TO THE GYROID WIRE NETWORK
}

\author{
RALPH M. KAUFMANN, SERGEI KHLEBNIKOV, \\ AND BIRGIT WEHEFRITZ-KAUFMANN
}

\begin{abstract}
Motivated by Harper Hamiltonians on skeletal graphs and their $C^{*}$-geometry, we study a certain class of graph Hamiltonians. These Hamiltonians can be thought of as a finite groupoid representation in separable Hilbert spaces. Here the groupoid is the path groupoid of a finite graph. Given such a setup, we consider the possible matrix versions of the Hamiltonian, which are indexed by the choice of a rooted spanning tree and an order of the vertices. The first result is that all the matrix representations are linked to each other via the conjugation action of a re-gauging groupoid.

We furthermore show that the symmetries of the underlying graph give rise to an action on the Hamiltonians of a group of extended symmetries. The new concept for the extension is to allow phase transformations on the vertices. In the commutative case, we prove that the extended symmetries act via a projective representation giving rise to isotypical decompositions and superselection rules.

We then apply these results to the PDG and honeycomb graphs using representation theory for projective groups and show that all the degeneracies in the spectra are consequences of these enhanced symmetries. This includes the Dirac points of the Gyroid and the honeycomb.
\end{abstract}

\section{INTRODUCTION}

Motivated by the synthesization of a novel material [1], we started an analysis of Harper Hamiltonians obtained from skeletal graphs [2]. Abstractly this situation is captured by graph Hamiltonians. Here the data is reduced to a finite graph $\bar{\Gamma}$ together with a separable Hilbert space for each vertex and a unitary morphism for each oriented edge, such that the inverse oriented edge corresponds to the inverse morphism. In this situation, as we derive, there is a Hamiltonian acting on the direct sum of all the Hilbert spaces and several matrix incarnations of it, which lie in $M_{k}(\mathscr{A})$. Here $k$ is the number of edges and $\mathscr{A}$ is the 
algebra generated by the morphisms corresponding to the loops on $\bar{\Gamma}$ with a fixed base point. Algebraically this setup is given by a groupoid representation in separable Hilbert spaces, as we explain in $§ 2.2$.

Physically the graph $\bar{\Gamma}$ arises as the quotient graph of a given (skeletal) graph $\Gamma \subset \mathbb{R}^{n}$ by a maximal translation group $L \simeq \mathbb{Z}^{n}$. Each edge of the quotient graph is decorated with a partial isometry operator of translation in the direction of any lift of that edge to the graph $\Gamma$. The Harper Hamiltonian is constructed from these isometries; in the simplest version (tight-binding approximation), it is simply the summation over them. This Hamiltonian along with the symmetries of the given material are the main input into the noncommutative geometry machine, which constructs a $C^{*}$-algebra that encodes relevant information about the system out of this data.

In the physical situation above, the algebra $\mathscr{A}$ depends on the choice of a magnetic field. Namely, if there is a constant $B$-field given by an anti-symmetric form $\Theta$ then $\mathscr{A}=\mathbb{T}_{\Theta}$-the noncommutative torus- as we showed in [2]. This is because in the presence of a $B$-field the translations cease to commute with the Hamiltonian (and with each other) and hence one enters the realm of noncommutative geometry. Here the translations together with the Hamiltonian generate a $C^{*}$-algebra, which we called the Bellissard-Harper algebra, $\mathscr{B}$. This approach is also useful in the commutative case of vanishing magnetic field. Here the $C^{*}$-algebra $\mathscr{B}$ corresponds to a compact Hausdorff space $X$, via the Gel'fand-Naimark theorem. This space is a branched cover of the $n$-torus $T^{n}=S^{1} \times \cdots \times S^{1}$, which is the space corresponding to $\mathbb{T}_{0}^{n}$. One way to view this cover is as the Eigenvalues of a family of Hamiltonians indexed over the torus (see $[2,3]$ ). Namely for every point in $t \in T^{n}$ there is a Hamiltonian $H(t) \in M_{k}(\mathbb{C})$, where $k$ is the number of vertices of $\bar{\Gamma}$. The fiber over a the point $t$ in the cover $\pi: X \rightarrow T^{n}$ is then the set of Eigenvalues of $H(t)$.

One main objective is to analyze and understand the branching behavior or stated otherwise the locus of degenerate Eigenvalues. The motivation is that in solid-state physics such degenerate Eigenvalues may lead to novel electronic properties, as is the case, for instance, with the Dirac points in graphene [4]. We have found two approaches to this question. The first is via singularity theory, which is contained in [3]. The second approach, which we take here is via group theory. Namely, we show that the symmetries of the underlying graph give rise to an action on the Hamiltonian of a group of extended symmetries. The key observations then are that (a) non-Abelian extended symmetry groups by themselves can force degeneracies via higher dimensional -i.e. $>1-$ irreducible representations and (b) any symmetries, also Abelian ones, 
give rise to super-selection rules. The latter ones can facilitate finding the spectrum considerably, since the Hamiltonian Eigenspace decomposition has to be compatible with the isotypical decomposition of the representation.

In order to find such symmetry groups, we start from the more general setup of graph Hamiltonians. Abstractly the setting can be understood as a groupoid representation as explained in $§ 2.2$. Here a vertex gets mapped to a separable Hilbert space $\mathscr{H}_{v}$ and the whole Hilbert space of the theory is the direct sum over all of these. The decoration of an edge is an isometry between the Hilbert space associated to the vertices of this edge. By fixing a rooted spanning tree, each edge corresponds to a loop and we can think of the isometries as just being unitary elements of a $C^{*}$-algebra $\mathscr{A}$, cf. $§ 2.2 .1$. In the physical applications this algebra will be the non-commutative torus $\mathbb{T}_{\Theta}^{n}$. In the commutative case, that of zero $B$-field, this is $\mathbb{T}^{n}=\mathbb{T}_{0}^{n}$, that is the $C^{*}$-algebra of continuous $\mathbb{C}$ valued functions on the $n$-torus $T^{n}$.

As worked out in [2] the fixing of a matrix version of the Hamiltonian from the $B$-field and the decorated graph $\bar{\Gamma}$, involves a choice of a rooted spanning tree $\tau$ of $\bar{\Gamma}$ and an order on the vertices. The choice of these pieces of data is, as we derive, what ultimately gives rise to the enhanced symmetry groups. The argument proceeds in several steps.

We first establish that the different matrix realizations of the Hamiltonian given by choosing different rooted spanning trees and orders are all linked by gauge-transformations - see Theorem 3.3. The specific gauge transformations that arise form a groupoid. This re-gauging groupoid $\mathcal{G}$ acts transitively on the set of all the matrix Hamiltonians obtained from the decorated graph. We furthermore show that the gauge transformations can be represented as conjugation with matrices. All the matrices here are matrices with values in $\mathscr{A}$. In the commutative case $(\S 3.3)$, we furthermore show that these matrices give a projective representation of the re-gauging groupoid. Just like in ordinary theory of projective representations this means that there is a bona fide representation of a central extension of this groupoid. In the case of our physical applications the extension is by $\mathbb{T}^{n}$.

If we fix a point $t \in T^{n}$ and evaluate the matrix Hamiltonians at $t$, the re-gauging actions give a groupoid representation in matrices $M_{k}(\mathbb{C})$. Now the stabilizer groups of a particular fixed Hamiltonian are the sought after enhanced symmetry groups.

For concrete applications this leaves the problem of identifying the points $t$ and the stabilizer groups or at least subgroups. To address the latter question, we establish that the automorphism group of $\bar{\Gamma}$ induces re-gaugings, by pushing forward the spanning tree and the 
orders. That is the symmetries of the graph give rise to re-gauging groupoid elements, actually a sub-groupoid. Going through the construction outlined above, we can restrict to this sub-groupoid and see that at a fixed point of the re-gauging action we get a projective representation of the stabilizer subgroup.

Furthermore, in order to identify points of $t$ where such enhanced symmetry groups can occur, we show that under certain assumptions, that hold in all cases of our initial physical interest, the classical symmetries of the graph $\bar{\Gamma}$ lift to an action on the base torus $T^{n}$ (Theorems 3.5 and 3.10). At points $t \in T^{n}$ with non-trivial stabilizer groups, we automatically get a projective representation of these stabilizer subgroups of the automorphism groups of the underlying graph, which commutes with the Hamiltonian. Hence we get isotypical decompositions, which can give us non-trivial information about the spectrum using the arguments (a) and (b) above.

We wish to point out that this approach is broader than that of considering classical symmetries of decorated graphs and in the commutative case generalizes the extensive analysis of [5], see $\S 3.1$ for details.

We apply all these considerations to the cases of the PDG wire networks and the honeycomb lattice; see $\S 4$. Our main result here is that at all degenerate points, which were analytically classified in [3], there is an enhanced symmetry group arising from graph automorphisms in the above way which forces the degeneracy.

Here the G-wire network corresponding to the double Gyroid which was our original motivator is the most interesting case. As shown in [3] there are exactly two points with triple degeneracies and two points with degeneracy $(2,2)$, that is two doubly degenerate Eigenvalues. The automorphism group of the graph is $\mathbb{S}_{4}$. The representation theory becomes very pretty in this case. There are two fixed points $(0,0,0)$ and $(\pi, \pi, \pi)$ on $T^{3}$ under the whole $\mathbb{S}_{4}$ action. The projective representation is just the ordinary representation of $\mathbb{S}_{4}$ by $4 \times 4$ permutation matrices at the point $(0,0,0)$ which is known to decompose into the trivial and a 3 dimension irreducible representation which forces the triple degeneracy. This result was also found by [5], where an initially different system was considered that results in the same spectrum.

At the point $(\pi, \pi, \pi)$ things become slightly more interesting. There is a projective representation of $\mathbb{S}_{4}$, but we can show that this projective representation corresponds to an extension which is isomorphic to the trivial extension. Hence after applying the isomorphisms we have a representation of $\mathbb{S}_{4}$ and there is a trivial and a 3-dim irrep, giving the second triple degeneracy. The elucidates the origin of the symmetry stated in [5]. Notice, that the classical symmetries of decorated graphs 
would only yield an $\mathbb{S}_{3}$ symmetry at this point, which cannot explain a triple degeneracy as there are no 3-dim irreps for this group.

Things really get interesting at the two points $( \pm \pi / 2, \pm \pi / 2, \pm \pi / 2)$. Here the stabilizer group is $A_{4}$. The projective representation gives rise to an extension which we show to be isomorphic to the non-trivial double cover $2 A_{4}$ of $A_{4}$ aka. $2 \mathrm{~T}$, the binary tetrahedral group or $S L(2,3)$. Using the character table we deduce that the representation decomposes into two 2-dim irreps forcing the two double degeneracies.

The identification of symmetries responsible for the degeneracies at $( \pm \pi / 2, \pm \pi / 2, \pm \pi / 2)$ is, as far as we know, new. We wish to point out that one absolutely needs the double cover as $A_{4}$ itself has no 2-dim irreps and hence the projective extension is essential.

We also use the fact that the diagonal of $T^{3}$ is fixed by a cyclic subgroup $C_{3}$ of $A_{4}$ in order to determine the spectrum analytically. Here we use the super-selection rules.

For the $\mathrm{D}$ and honeycomb case, we show that the degenerate points which are well known in the honeycomb case and were computed for $\mathrm{D}$ in [6] are all detected by enhanced symmetries. These however yield Abelian representations and hence we have to use the arguments of the type (b), that is super-selection rules, to show that the Eigenvalues are degenerate over these points.

\section{General Setup}

We fix a unital $C^{*}$-algebra $\mathscr{A}$. In our applications this will be the noncommutative torus $\mathbb{T}_{\Theta}^{n}$. Although eventually $\mathscr{A}$ will be commutative - in particular $\mathbb{T}_{0}^{n}$ in the applications - at the start, we do not assume that $\mathscr{A}$ is commutative. We develop the theory in the general noncommutative setting as far as possible for possible further applications.

The basic object of study will be a $C^{*}$-algebra $\mathscr{B}$ called the BellissardHarper algebra generated by a symmetry $C^{*}$-algebra $\mathscr{A}$ and a Hamiltonian $H$. The Hamiltonian and the algebra $\mathscr{A}$ are given by a groupoid representation. This is in a sense inverse to [2], where the algebra $\mathscr{A}$ given by magnetic translations along a lattice was a fundamental a priori datum. However, under certain minimality assumptions it is fully recoverable.

Ultimately we will present results about the symmetries of the Hamiltonian in the commutative case. The reason that these exists can be traced back to a projective action of a re-gauging groupoid. Said groupoid already exists in the noncommutative case. 
We will first give the algebraic setting and introduce the first mentioned groupoid representation. The readers not interested in this underlying layer can skip to the section $§ 2.2 .3$ in which we give the matrix versions of the Hamiltonian.

Before delving into the data and axioms of the general theory, we will briefly review the geometric constructions of $[2,6]$ which are our main motivator. Again this section can be skipped if one wishes to concentrate on the more formal aspects, although it illustrates the way these structures arise and are interlinked in a very good manner.

2.1. The geometric setup of wire networks. The PDG examples are based on the unique triply periodic CMC surfaces where the skeletal graph is symmetric and self-dual. Physically, in the $\mathrm{P}$ (primitive), D (diamond) and G (Gyroid) case, one starts with a "fat" or thick version of this surface, which one can think of as an interface. A solidstate realization of the "fat" Gyroid aka. double Gyroid has recently been synthesized on the nano-scale [1]. The structure contains three components, the "fat" surface or wall and two channels. Urade et al. [1] have also demonstrated a nanofabrication technique in which the channels are filled with a metal, while the silica wall can be either left in place or removed. This yields two wire networks, one in each channel. The graph we consider and call Gyroid graph is the skeletal graph of one of these channels. The graph Hamiltonian we construct algebraically below is the tight-binding Harper Hamiltonian for one channel of this wire network. The $2 \mathrm{~d}$ analogue is the honeycomb lattice underlying graphene. Graph theoretically the quotient graph for the honeycomb is the $2 \mathrm{~d}$ version of that of the D surface, but as we showed, the behavior, such as the existence of Dirac points [3], is more like that of the Gyroid surface.

To formalize the situation, we regard the skeletal graph of one channel as an embedded graph $\Gamma \subset \mathbb{R}^{n}$. The crystal structure gives a maximal translational symmetry group $L$ which is a mathematical lattice, i.e. isomorphic to $\mathbb{Z}^{n}$, s.t. $\bar{\Gamma}:=\Gamma / L$ is a finite connected graph. The vertices of this quotient graph are the elements in the primitive cell.

The Hilbert space $\mathscr{H}$ for the theory is $\ell^{2}(V(\Gamma))$, where $V(\Gamma)$ are the vertices of $\Gamma$. This space splits $\bigoplus_{v \in V(\bar{\Gamma})} \mathscr{H}_{v}$ where for each vertex $v \in V(\bar{\Gamma}), \mathscr{H}_{v}=\ell^{2}\left(\pi^{-1}(v)\right)$ where $\pi: \Gamma \rightarrow \bar{\Gamma}$ is the projection. All the spaces $\mathscr{H}_{v}$ are separable Hilbert spaces and hence isomorphic. Furthermore if $\vec{e}$ is a directed edge from $v$ to $w$ in $\bar{\Gamma}$ then it lifts uniquely as a vector to $\mathbb{R}^{n}$, which we denote by the same name. Moreover for each such vector there is a naturally associated translation operator 
$T_{\vec{e}}: \mathscr{H}_{v} \rightarrow \mathscr{H}_{w}$, by the usual action of space translations on functions. We also allow for a constant magnetic field $B=2 \pi \hat{\Theta}$ where $\hat{\Theta}=\sum \theta_{i j} d x_{i} \wedge d x_{j}$ is a constant 2 -form given by the skew-symmetric matrix $\Theta=\left(\theta_{i j}\right)_{i j}$. If $\Theta \neq 0$ then the translations become magnetic translations or Wannier operators $U_{\vec{e}}: \mathscr{H}_{v} \rightarrow \mathscr{H}_{w}$; see e.g. [7]. These operators are still unitary and give partial isometries when regarded on $\mathscr{H}$ via projection and inclusion, which we again denote by the same letter.

The Harper Hamiltonian is then defined as

$$
H=\sum_{e \in E(\bar{\Gamma})}\left(U_{\vec{e}}+U_{\overleftarrow{e}}\right)
$$

where the sum runs over all edges of $\bar{\Gamma}$ and given an edge $e, \vec{e}$ and $\overleftarrow{e}$ are its two orientations. Notice that $U_{\vec{e}}^{*}=U_{\overleftarrow{e}}$.

Likewise $L$ acts by magnetic translations. If $\vec{\lambda}$ is a vector in $L$ then $U_{\rightarrow}$ sends each $\mathscr{H}_{v}$ to itself and the diagonal action gives an action on $\hat{\mathscr{H}}$. Since $L$ is a lattice, the representation it generates is given by $n$ linearly independent unitaries. The commutation relations among the Wannier operators amount to the fact that the representation is nothing but a copy of $\mathbb{T}_{\Theta}^{n}$, the non-commutative $n$-torus, see [2] or [7]. This gives rise to a "diagonal" representation $\mathbb{T}_{\Theta}^{n} \rightarrow B(\mathscr{H})$ via the direct sum decomposition. Here, as usual, $B(\mathscr{H})$ is the $C^{*}$-algebra of bounded operators on $\mathscr{H}$. This representation and $H$ by definition generate the Bellissard-Harper $C^{*}$-algebra $\mathscr{B}$.

To do calculations it is convenient, however, to have the Hamiltonian in a matrix form. This is achieved as follows. Fix a rooted spanning tree $\tau$ of $\bar{\Gamma}$. This is by definition a contractible subgraph which contains all the vertices of $\bar{\Gamma}$ and in addition one of the vertices is declared the root; denote it by $v_{0}$. In order to obtain a proper matrix, we also need to fix an order $<$ on all the vertices, where we insist that the root is the first in this order. For each vertex $v$ of $\bar{\Gamma}$ there is a unique shortest path in $\tau$ to $v_{0}$. This defines a choice of fixed isomorphism $U_{v v_{0}}^{\tau}: \mathscr{H}_{v_{0}} \rightarrow \mathscr{H}_{v}$ by translations along the edge path, see $\S 3.2$ for details.

Using these isomorphisms, we can conjugate the Harper Hamiltonian to a matrix Harper Hamiltonian $H_{\tau, v_{0},<}$ or $H_{\tau,<}$ for short which acts on $\bigoplus_{v \in V(\bar{\Gamma})} \mathscr{H}_{v_{0}}=\mathscr{H}_{v_{0}}^{k}$ if $k=|V(\bar{\Gamma})|$. That is $H_{\tau,<}=U^{*} H U \in M_{k}\left(\mathbb{T}_{\Theta}^{n}\right)$ where $U=\bigoplus_{v \in V(\bar{\Gamma})} U_{v v_{0}}^{\tau}$ and the matrix entries which belong to $\mathbb{T}_{\Theta}$ act on $\mathscr{H}_{v_{0}}$ again via translation. The "diagonal" action of $\mathbb{T}_{\Theta}$ on $\mathscr{H}$ also pulls back to $\mathscr{H}_{v_{0}}^{k}$ via $U$ and yields a faithful matrix representation 
of $\mathbb{T}_{\Theta}$. This algebra and $H_{\tau,<}$ generate a faithful matrix representation of $\mathscr{B}$.

Although different choices lead to isomorphic subalgebras of $M_{k}\left(\mathbb{T}_{\Theta}\right)$, the concrete algebras depend on the choice of order and spanning tree. As we show, changing the underlying data gives rise to a re-gauging groupoid which ultimately gives rise to the symmetries forcing degeneracies in the spectrum over certain points.

\subsection{Groupoid graph representations in separable Hilbert spaces.}

The abstraction of the setting above is given by the following data. Given a finite graph $\bar{\Gamma}$ we define a groupoid representation of $\bar{\Gamma}$, an association of a separable Hilbert space $\mathscr{H}_{v}$ for each $v \in V(\bar{\Gamma})$ and an isometry $U_{\vec{e}}: \mathscr{H}_{v} \rightarrow \mathscr{H}_{w}$ for each directed edge $\vec{e}$ from $v$ to $w$. This data indeed determines a unique functor from the path groupoid of $\bar{\Gamma}$ to the category of separable Hilbert spaces. The path groupoid of $\bar{\Gamma}$ is category whose objects are the vertices of $\bar{\Gamma}$ and whose morphisms are generated by the oriented edges, where the inverse of a morphism given by $\vec{e}$ is the one given by $\overleftarrow{e}$. Notice, that we are looking at the morphisms generated by the oriented edges, this means that $\operatorname{Hom}(v, w)$ is the set of paths along oriented edges from $v$ to $w$ modulo the relation that going back and forth along an edge is the identity.

In particular, this lets one view $\pi_{1}\left(\bar{\Gamma}, v_{0}\right)$ in two equivalent fashions. First as the topological $\pi_{1}$ of the realization of a graph, and secondly as the group $\operatorname{Hom}\left(v_{0}, v_{0}\right)=\operatorname{Aut}\left(v_{0}\right)$ where Hom are the morphisms in the groupoid.

We let $\mathscr{A}$ be the abstract $C^{*}$-algebra $\pi_{1}$ generates in $B(\mathscr{H})$ via the representation. This is a bit subtle, as the concrete algebra depends on the choice of $v_{0}$, but of course any two choices give isomorphic algebras.

Given a groupoid representation as above, set $\mathscr{H}=\bigoplus_{v \in V(\bar{\Gamma})} \mathscr{H}_{v}$ and define $H$ by equation (1). Fix a rooted spanning tree and an order on the vertices. Then again, we can identify $\mathscr{H} \simeq \mathscr{H}_{v_{0}}^{k}$ via a unitary $U$ just as above.

After having fixed an initial spanning tree the matrix Hamiltonian has a different description. To each edge $\vec{e}$ from $v$ to $w$, we can associate $w t(\vec{e}):=U_{v_{0} v}^{\tau} U_{\vec{e}} U_{v v_{0}}^{\tau} \in U(\mathscr{A})$. That is, we can regard $\bar{\Gamma}$ as having a weight function on ordered edges with weights in $U(\mathscr{A})$. If $e$ is an edge of $\tau$ then with this definition $w t(\vec{e})=w t(\overleftarrow{e})=1$

An alternative way of viewing this data is as a certain type of quiver representation, we will comment on this more below.

2.2.1. Fundamental group and Euler Characteristic. For any finite graph $\bar{\Gamma}$ the Euler Characteristic is $\chi(\bar{\Gamma})=|V(\bar{\Gamma})|-|E(\bar{\Gamma})|=1-b_{1}$ where $b_{1}$ 
is the rank of $H_{1}(\bar{\Gamma})$ which is the same as the "number of loops". More precisely, if $\bar{\Gamma}$ is connected, $\pi_{1}(\bar{\Gamma})=\mathbb{F}_{b_{1}}$ that is the free group in $b_{1}$ generators. In the examples above $b_{1}=n$, the rank of $L$. One way to view a rooted spanning tree $\left(\tau, v_{0}\right)$ is to think of it as fixing a base point $v_{0}$ and a set of symmetric generators/basis for $\pi_{1}\left(\bar{\Gamma}, v_{0}\right)=\operatorname{Hom}\left(v_{0}, v_{0}\right)$. Topologically after contracting the spanning tree one is left with a wedge of $S^{1}$ s. There are $b_{1}$ of these one for each non-contracted edge. Each simple loop around one of the $S^{1}$ s gives a generator. Picking only one generator per loop gives a basis.

Without doing the contraction the correspondence on $\bar{\Gamma}$ itself is given by all the (ordered) edges not contained in $\tau$. To each such ordered edge $\vec{e}$ from $v$ to $w$, we associate the loop starting at $v_{0}$ going along $\tau$ to $v$ then traversing $\vec{e}$ to $w$ and afterwards returning to $v_{0}$ along $\tau$. Again picking both orientations gives a symmetric set of generators of the free group while picking only one orientation per edge fixes a basis. Any edge in the spanning tree corresponds to the unit, the constant loop.

In this language, the weight function $w t$ is a representation of $\pi_{1}$ lifted to the edges of the graph by the above correspondence. Thus, as long as the base points $v_{0}$ stays fixed, the changes of spanning tree thus can be viewed as changing of a basis of $\pi_{1}\left(\bar{\Gamma}, v_{0}\right)$. If $v_{0}$ moves, say to $v_{0}^{\prime}$, then, as usual, any path from $v_{0}^{\prime}$ to $v_{0}$ gives an isomorphism taking $\pi_{1}\left(\bar{\Gamma}, v_{0}^{\prime}\right)$ to $\pi_{1}\left(\bar{\Gamma}, v_{0}\right)$. Both types of isomorphisms will play a role later in the symmetry group actions.

2.2.2. Non-degeneracy and toric non-degeneracy. We call a groupoid representation non-degenerate, if the images of basis of the free group given by the construction above are independent unitary generators of $\mathscr{A}$ and call it toric non-degenerate if $\mathscr{A}$ is isomorphic to the noncommutative torus $\mathbb{T}_{\Theta}^{b_{1}}$.

Notice that if $\mathscr{A}$ is commutative and non-degenerate, then $\mathscr{A} \simeq \mathbb{T}^{b_{1}}$, the $C^{*}$ algebra of the torus of dimension $b_{1}$.

2.2.3. Hamiltonian and $\mathscr{A}$ from a weighted graph. After the preparation step above, we end up with a Hamiltonian and symmetry algebra $\mathscr{A}$ as above. One can also choose this as the starting point, which is what we will do now.

Fix a finite connected graph $\bar{\Gamma}$, a rooted spanning tree $\tau$ of $\bar{\Gamma}$, an order $<$ of the vertices of $\Gamma$ such that the root of $\tau$ is the first vertex, a unital $C^{*}$ algebra $\mathscr{A}$, and a morphism $w t:\{$ Directed edges of $\Gamma\} \rightarrow \mathscr{A}$ which satisfies 
(1) $w t(\vec{e})=w t(\overleftarrow{e})^{*}$ if $\vec{e}$ and $\overleftarrow{e}$ are the two orientations of an edge $e$.

(2) $w t(\vec{e})=1 \in \mathscr{A}$ if the underlying edge $e$ is in the spanning tree.

In general, if $w t$ is as above and it satisfies the first condition, we will call it a weight function (with values in $\mathscr{A}$ ) and if it satisfies both conditions, a weight function compatible with the spanning tree.

Fix a separable Hilbert space $\mathscr{H}_{v_{0}}$. By Gel'fand Naimark representability we realize $\mathscr{A} \subset B\left(\mathscr{H}_{v_{0}}\right)$ and we shall use this representation.

We shall also postulate that $\mathscr{A}$ is minimal, that is that it is the $C^{*}$-algebra generated by the $w t(\vec{e})$ where $\vec{e}$ runs through the directed edges of $\bar{\Gamma}$. This makes the terminology of $\S 2.2 .2$ applicable. Also, we see that this is again just a lift of a representation of $\pi_{1}\left(\bar{\Gamma}, v_{0}\right)$ to the edges of $\bar{\Gamma}$ using the spanning tree $\tau$.

Given this data, let $k$ be the number of vertices of $\Gamma$. We will enumerate the vertices $v_{0}, \ldots, v_{k-1}$ according to their order; $v_{0}$ being the root. Given this data, the Hamiltonian $H=H(\Gamma, \tau,<, w)$ is the $k \times k$ matrix whose entries are in $\mathscr{A}$

$$
H_{i j}=\sum_{\text {directed edges } \vec{e} \text { from } v_{i} \text { to } v_{j}} w t(\vec{e})
$$

We now consider $\mathscr{H}:=\mathscr{H}_{0}^{k}$ then $H=\left(H_{i j}\right)_{i j}$ is naturally a matrix in $M_{k}(\mathscr{A})$.

We see that the weighted graph encodes both the Hamiltonian and the symmetry algebra $\mathscr{A}$.

Remark 2.1. In the general noncommutative case, this is not quite enough for the whole theory, as we do not recover the action of $\mathscr{A}$ on $\mathscr{H}$ and the connection between the action of $H$ and that of $\mathscr{A}$. Recall that the action of $\mathscr{A}$ on $\mathscr{H}=\mathscr{H}_{v_{0}}^{k}=\bigoplus_{v \in V(\bar{\Gamma})} \mathscr{H}_{v_{0}}$ is given on each summand $\mathscr{H}_{v_{0}}$ corresponding to $v$ by pulling back the action from $\mathscr{H}_{v}$. That is, the action is a conjugated action.

In the commutative case this is not an issue as the representation is exactly the diagonal representation.

2.2.4. Geometry in the Commutative case. If $\mathscr{B}$ is commutative (and hence also $\mathscr{A}$ ), as for instance if $\Theta=0$, then there is a geometric version of these algebras which can be understood as the spectra of a family of Hamiltonians over a base. We have the following inclusion of commutative $C^{*}$ algebras $i: \mathscr{A} \hookrightarrow \mathscr{B}$, by Gel'fand Naimark this 
gives us a surjection of compact Hausdorff spaces $^{1} \pi: X \rightarrow B$ where $C(B) \simeq \mathscr{A}$ and $C(X) \simeq \mathscr{B}$. The correspondence is given via characters. Namely a character is a $C^{*}$-homomorphism $\chi: \mathscr{A} \rightarrow \mathbb{C}$. The characters are by definitions the points of $B$. Vice-versa any point $t \in B$ determines a character $e v_{t}: C(B) \rightarrow \mathbb{C}$ via evaluation. That is any $f \in C(B)$ is sent to $f(t) \in C$. Given a character $\chi$ on $\mathscr{A}$, we can lift it to a $C^{*}$-morphism $\hat{\chi}: M_{k}(\mathscr{A}) \rightarrow M_{k}(\mathbb{C})$ by applying it in each matrix entry.

Thus any point $t \in B$ represented by the character $\chi$ determines a Hamiltonian $\hat{\chi}(H) \in M_{k}(\mathbb{C})$ via $\hat{\chi}$.

$$
(\hat{\chi}(H))_{i j}=\chi\left(H_{i j}\right)
$$

Thus we get a family of Hamiltonians $H(t)$ parameterized over the base. One can furthermore check, see [2], that $\pi$ is a branched cover over $\mathscr{A}$ with $\pi^{-1}(t)=\operatorname{spec}(H(t))$.

2.3. Gauge group action. We stress that $H$ as a matrix depends on all of this data. In the geometric situation of [2] this data was necessary to obtain a true matrix from the original Harper Hamiltonian. In the commutative case, changing the data does not change the Eigenvalues as they do not depend on the chosen isomorphisms.

\section{Symmetries}

In order to deal with the symmetries of the graph it is helpful to first fix the notation as sometimes questions become subtle.

3.1. Classical Symmetries. A graph $\Gamma$ is described by a set of vertices $V_{\Gamma}$ and a set of edges $E_{\Gamma}$ together with incidence relations $\partial$ where for each edge $e, \partial(e)=\{v, w\}$ is the unordered set of the two vertices it is incident to. A directed edge is given by an order on this pair. Hence for each edge $e$ there are two ordered edges by the orders $(v, w)$ and by $(w, v)$. We usually denote these two edges by $\vec{e}$ and $\overleftarrow{e}$. The set of all oriented edges is called $E_{\Gamma}^{o r}$.

An isomorphism $\phi$ of two graphs $\Gamma$ and $\Gamma^{\prime}$ is a pair of bijections $\left(\phi_{V}, \phi_{E}\right) \phi_{V}: V_{\Gamma} \rightarrow V_{\Gamma^{\prime}}$ and $\phi_{E}: E_{\Gamma} \rightarrow E_{\Gamma^{\prime}}$. The compatibility is that the incidence conditions are preserved, i.e.: if $\partial(e)=\{v, w\}$ then $\partial\left(\phi_{E}(v)\right)=\left\{\phi_{V}(v), \phi_{V}(w)\right\}$. Notice the $\phi$ also induces a map of oriented edges, the orientation of the edge $\phi_{E}(e)$ given by $\left(\phi_{V}(v), \phi_{V}(w)\right)$, if the orientation of $e$ is $(v, w)$.

We will treat isomorphism classes of graphs from now on. Fixing an isomorphism class of a graph still allows for automorphisms. These are

\footnotetext{
${ }^{1}$ Both $\mathscr{A}$ and $\mathscr{B}$ are unital
} 


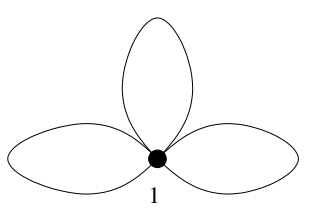

$\mathrm{P}$

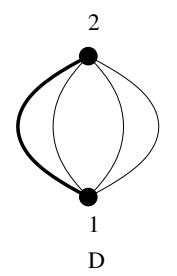

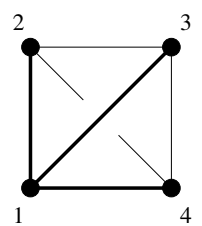

G

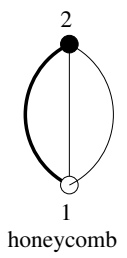

Figure 1. The graphs P, D and G and honeycomb together with the preferred spanning tree and order.

given as follows. Fix a representative of $\Gamma$ then an automorphism is a pair of compatible maps $\left(\phi_{V}, \phi_{E}\right) \phi_{V}: V_{\Gamma} \rightarrow V_{\Gamma}$ and $\phi_{E}: E_{\Gamma} \rightarrow E_{\Gamma}$.

Example 3.1. Let us illustrate this for the graphs corresponding the PDG and honeycomb cases which are given in Figure 1; see $\S 2.1$ for details about the corresponding wire networks. We fix once and for all an isomorphism class of these graphs and then consider their automorphisms using the representatives given in that figure..

For the $\mathrm{P}$ case there is only one vertex hence $\phi_{V}=i d$ is the only possibility. However there is an $\mathbb{S}_{3}$ action permuting the three loop edges.

The D graph has the possibility of switching the two vertices and freely permuting the three edges. This gives the automorphism group $\mathbb{Z} / 2 \mathbb{Z} \times \mathbb{S}_{3}$. The honeycomb similarly has automorphism group $\mathbb{Z} / 2 \mathbb{Z} \times$ $\mathbb{S}_{3}$.

For the Gyroid, there is an $\mathbb{S}_{4}$ worth of potential choices for $\phi_{V}$. Now all these choices extend uniquely to the edges, since there is exactly one edge between each distinct pair of vertices and hence the symmetry group is exactly $\mathbb{S}_{4}$.

3.1.1. Pushing forward Spanning Trees. Given a pair $(\Gamma, \tau)$ of a graph and a rooted spanning tree, we define the action of isomorphisms and automorphisms by push-forward. That is an isomorphism between $(\Gamma, \tau)$ and $\left(\Gamma^{\prime}, \tau^{\prime}\right)$ is an isomorphism from $\Gamma$ to $\Gamma^{\prime}$ such that that $\phi_{V}$ maps the root of $\tau$ to the root of $\tau^{\prime}$ and $\phi$ restricted to $\tau$ is an isomorphism onto $\tau^{\prime}$.

If we have not already specified a spanning tree on $\Gamma^{\prime}$ we can extend any isomorphism $\phi$ from $(\Gamma, \tau)$ to it by push-forward. This means that we push-forward all the vertices and the edges of the spanning tree $\tau$ to $\Gamma^{\prime}: E_{\tau^{\prime}}:=\phi_{E}\left(E_{\tau}\right)$ and likewise push-forward the root.

In particular, $A u t(\Gamma)$ acts on the set of spanning trees of a fixed graph $\Gamma$. This action is not transitive in general and may have fixed points. 
Example 3.2. In the cases of PDG and the honeycomb, it is a transitive action.

For the $G$ graph the action is not fixed point free, there is an $\mathbb{S}_{3}$ subgroup fixing a given spanning tree.

For the $\mathrm{P}$ graph the action is fixed point free, while for the $\mathrm{D}$ and the honeycomb although the action is transitive, there are again stabilizers. For the honeycomb the group fixing a spanning tree is the $\mathbb{S}_{2}=\mathbb{Z} / 2 \mathbb{Z}$ interchanging the two other edges, with both vertices fixed, while in the $\mathrm{D}$ case it is an $\mathbb{S}_{3}$ action interchanging the non spanning tree edges.

In the triangle graph, with one edge doubled, we can see that there are two orbits. One where the two non-doubled edges give the spanning tree, there is an $\mathbb{S}_{2}$ stabilizer interchanging the non-root vertices, one where one of the doubled edges is in the spanning tree, there is no stabilizer in this orbit.

3.1.2. Orders, Weight Functions and Isomorphisms. Also if there is an order on all the vertices then the isomorphisms are asked to be compatible with this order and auto- and isomorphisms can be extended by pushing forward the order.

A final piece of data, which we utilize is a $C^{*}$ algebra valued map $w t: E_{\Gamma}^{o r} \rightarrow \mathscr{A}$, such that $w t(\overleftarrow{e})=w t(\vec{e})^{*}$. In our case of interest $\mathscr{A}=\mathbb{T}_{\Theta}^{n}$, and even $\mathbb{T}_{0}^{n}$ later, but for now we will keep the abstract setting.

Notice that a given weight function might not be compatible with the new, pushed-forward spanning tree, since it does not necessarily satisfy the condition (2).

The idea of re-gauging is to fix condition (2) by passing to an equivalent, re-gauged weight function.

One way to see the failure to preserve the compatibility condition is view the change of spanning tree as a change of basis of $\pi_{1}$ or rather an isomorphism of $\pi_{1} \mathrm{~s}$ via the path-groupoid. This point of view is at the basis of the proof of Theorem 3.5 below.

3.1.3. Classical vs. extended symmetries. There is another natural choice of iso- or automorphism for graphs with weight functions. Here one would postulate that the weight functions are compatible. Weight functions naturally pull back via $\phi_{E}$, that is $\phi_{E}^{*}(w)(\vec{e})=w t\left(\phi_{E}(\vec{e})\right)$. Using that $\phi_{E}$ is an isomorphism, one can push-forward by pulling back along $\phi_{E}^{-1}$.

One could call these symmetries classical symmetries of the weighted graph. These are the kinds of symmetries that were for instance considered by [5]. 
We will consider symmetries of the underlying graph not of the weighted graph. The weights are fixed by re-gauging. One way to phrase this is that is we utilize an extended symmetry group which allows for phase factors at the vertices. The details are given below.

3.2. Gauging. One of the key observations of [2] (see §2.1) is that there are several equivalent matrix versions of the Harper Hamiltonian. This applies also to the case of matrix Hamiltonians from weighted graphs.

Starting from the groupoid representation we obtain $H$ as an endomorphism of $\mathscr{H}=\bigoplus_{v \in V_{\bar{\Gamma}}} \mathscr{H}_{v}$, where the entries are partial isometries $U_{v w}: \mathscr{H}_{w} \rightarrow \mathscr{H}_{v}$, with $U_{w v}=U_{v w}^{-1}$. By fixing a rooted spanning tree $\tau$, one can derive matrix versions, which act on $\bigoplus \mathscr{H}_{v_{0}}$, where $v_{0}$ is the chosen root. There is one more caveat: in order to have a true matrix, we also need to fix an order on all the vertices, which we fix to always start with $v_{0}$. Let $v_{i}$ be the i-the vertex in this enumeration. Then we obtain a matrix $H_{\tau,<}$ by using the isomorphisms $U_{v_{i} v_{0}}^{\tau}$ which are defined as follows. Let $v_{0}, w_{1}, \ldots, w_{k}, v_{i}$ be the sequence of vertices along the unique shortest path from $v_{0}$ to $v_{i}$ in $\tau$, then

$$
U_{v_{i} v_{0}}^{\tau}=U_{v_{i} w_{k}} U_{w_{k} w_{k-1}} \ldots U_{w_{2} w_{1}} U_{w_{1} v_{0}}
$$

and $U_{v_{0} v_{i}}^{\tau}=\left(U_{v_{i} v_{0}}^{\tau}\right)^{*}$. Given the choice of $(\tau,<)$, we get the corresponding matrix Hamiltonian as

$$
H_{\tau,<}: \bigoplus_{i=1}^{k} \mathscr{H}_{v_{0}} \stackrel{\bigoplus_{i} U_{v_{0} v_{i}}^{\longrightarrow}}{\longrightarrow} \bigoplus_{i} \mathscr{H}_{v_{i}}=\mathscr{H} \stackrel{H}{\longrightarrow} \mathscr{H}=\bigoplus_{i} \mathscr{H}_{v_{i}} \stackrel{\bigoplus_{i} U_{v_{i} v_{0}}^{\tau}}{\longrightarrow} \bigoplus_{i} \mathscr{H}_{v_{0}}
$$

Of course, all the $\mathscr{H}_{\tau,<}$ are all equivalent, although not canonically. For the equivalence one has to choose a path from one root to the other. We will exploit this fact extensively below.

In the language we have set up, we get a weight function $w t(\vec{e})=$ $U_{v_{0} v}^{\tau} U_{v w} U_{w v_{0}}^{\tau}$ and $H$ is then the graph Hamiltonian of the representation which associates to each vertex the Hilbert space $\mathscr{H}_{v_{0}}$ and to each directed edge $\vec{e}$ the endomorphism $w t(\vec{e})$.

We will now consider the relationship between the different matrices $H_{\tau,<}$ and $H_{\tau^{\prime},<^{\prime}}$. Notice that although they are always matrices in $M_{k}(\mathscr{A})$, they represent $H$ via different isomorphisms. More precisely, the first and last arrow in the composition will differ.

There are basically three situations: First, $\tau=\tau^{\prime}$ as rooted trees and only the order changes. Secondly, $\tau=\tau^{\prime}$ but $v_{0} \neq v_{0}^{\prime}$ and thirdly, the trees just do not coincide, i.e. at least one edge is different. In the first case the isomorphisms are simply changed by permutations 
of the factors $\mathscr{H}_{v_{0}}$. This means that the difference between the two matrix Hamiltonians is simply conjugation by $M_{\sigma}$ where this is the standard permutation matrix. Since $v_{0}$ is fixed to be the first element, the permutation actually lies in the subgroup $\mathbb{S}_{k-1} \subset \mathbb{S}_{k}$ fixing the first element. $\mathbb{S}_{k-1}$ then acts simply transitively on the orders.

In the second and third case, the situation is more complicated. Of course the second type of change is related to an action of $\mathbb{S}_{k}$, but things are not that simple, since there is a change of the space that $H_{\tau^{\prime},<^{\prime}}$ acts on. If the tree moves, then we have to also change the weight functions to make them compatible with the new spanning tree.

Taking the point of view of $\pi_{1}$ the first type of transformation is just a permutation of the basis. Keeping the base point, viz. the root fixed, we get a change of basis, but when we move the base point, we get an isomorphic group. In doing this, we effectively use the path groupoid. In pure matrix language, we will show in the next section that the matrices change by a re-gauging, which we can realize by conjugation. These re-gaugings are governed by a groupoid, the regauging groupoid.

3.2.1. Gauging in groupoid representations. As discussed in 2.2 the Harper Hamiltonian before fixing a spanning tree can be thought of as a certain type of groupoid representation.

For such representation, we can re-gauge it by acting with automorphisms of the $\mathscr{H}_{v}$, that is the group $\left.\times_{v \in \Gamma} A u t\left(\mathscr{H}_{v}\right)\right)$. Here $\mathscr{H}_{v}$ is the Hilbert space associated to $v$ and $U_{\vec{e}}$ is the morphism $\mathscr{H}_{v} \rightarrow \mathscr{H}_{w}$ given by the representation where $\vec{e}$ runs from $v$ to $w$. Picking an element $\phi$ in this group is the same as the assignment $v \mapsto \phi(v) \in \operatorname{Aut}\left(\mathscr{H}_{v}\right)$ this means that $U_{\vec{e}}$ gets re-gauged to $\phi(w) U_{\vec{e}} \phi^{-1}(v)$. This gives an equivalent representation.

In our situation, since we have Hilbert spaces, we can look at unitary equivalences and restrict the automorphisms to be unitary. Also, choosing an identification of all the isomorphic separable Hilbert spaces $\mathscr{H}_{v}$ with some fixed $\mathscr{H}_{v_{0}}$ we can take the re-gaugings to live in the unitary operators on $\mathscr{H}_{v_{0}}$.

\subsubsection{Spanning tree re-gauging.}

Theorem 3.3. Given two ordered rooted spanning trees $(\tau,<)$ and $\left(\tau^{\prime},<^{\prime}\right)$ there is a matrix $M \in M_{k}(\mathscr{A})$ with $M M^{*}=M^{*} M=i d$ such that $M H_{\tau,<} M^{*}=U_{v_{0} v_{0}^{\prime}}^{\tau} H_{\tau^{\prime},<^{\prime}} U_{v_{0}^{\prime} v_{0}}^{\tau}$ 
Proof. Consider the commutative diagram :

(6)

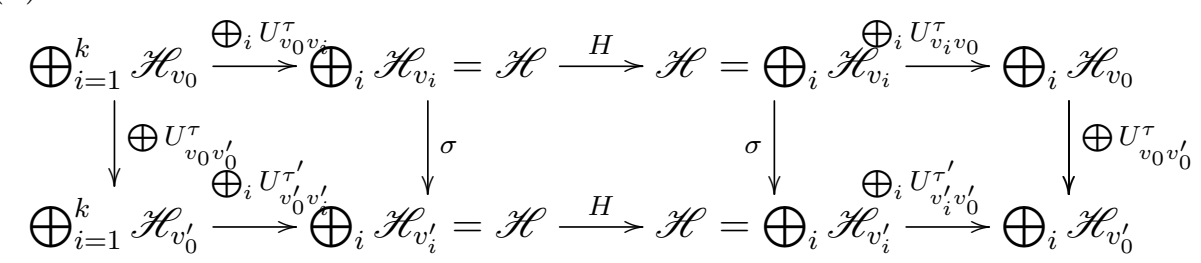

We see that if $i^{\prime}=\sigma(i)$ and $j^{\prime}=\sigma(j)$ so that $v_{i^{\prime}}^{\prime}=v_{i}$ :

$$
\begin{aligned}
& \left(H_{\tau^{\prime},<^{\prime}}\right)_{i^{\prime} j^{\prime}}=U_{v_{0}^{\prime} v_{i^{\prime}}^{\prime}}^{\tau^{\prime}} H_{v_{i^{\prime}} v_{j^{\prime}}^{\prime}} U_{v_{j^{\prime}}^{\prime} v_{0}^{\prime}}^{\tau^{\prime}} \\
= & U_{v_{0}^{\prime} v_{0}}^{\tau}\left(U_{v_{0} v_{0}^{\prime}}^{\tau} U_{v_{0}^{\prime} v_{i^{\prime}}^{\prime}}^{\tau^{\prime}} U_{v_{i} v_{0}}^{\tau}\right)\left(U_{v_{0} v_{i}}^{\tau} H_{v_{i} v_{j}} U_{v_{j} v_{0}}^{\tau}\right)\left(U_{v_{0} v_{j}}^{\tau} U_{v_{j^{\prime}}, v_{0}^{\prime}}^{\tau^{\prime}} U_{v_{0}^{\prime} v_{0}}^{\tau}\right) U_{v_{0} v_{0}^{\prime}}^{\tau} \\
= & U_{v_{0}^{\prime} v_{0}}^{\tau} \phi_{i^{\prime}}^{*}\left(H_{\tau,<}\right)_{i j} \phi_{j^{\prime}} U_{v_{0} v_{0}^{\prime}}^{\tau}
\end{aligned}
$$

With $\phi_{j^{\prime}}=U_{v_{0} v_{j}}^{\tau} U_{v_{j^{\prime}}, v_{0}^{\prime}}^{\tau^{\prime}} U_{v_{0}^{\prime} v_{0}}^{\tau} \in \mathbb{T}_{\Theta}^{n}$. So that if $\Phi=\operatorname{diag}\left(\phi_{i^{\prime}}\right)$ and $M_{\sigma}$ is the permutation matrix of $\sigma$ then $M=\Phi^{*} M_{\sigma^{-1}}$ and $M^{*} M=i d$.

We choose to place $M$ on the left of the Hamiltonian so as to get a left action.

Proposition 3.4. The matrix $\Phi$ above can be expressed as a re-gauging, that is there is an element in the gauge group whose matrix representation is $\Phi$.

Proof. Unraveling the definition given in equation (4), we can express the matrix $\Phi$ by the following iterative procedure. We start at the root of $\tau^{\prime}$ and choose $\phi\left(v_{0}^{\prime}\right)=i d$. Assume we have already assigned weights to all vertices at distance $i$ from $v_{0}^{\prime}$ and let $w$ be a vertex at distance $i+1$. Then there a unique $v$ at distance $i$ which is connected to $W$ along a unique directed edge $\vec{e}$ fof the spanning tree $\tau^{\prime}$. Set $\phi(w)=$ $w t(\vec{e}) \phi(v) \in U(\mathscr{A})$. If $\sigma \in \mathbb{S}_{k}$ is the unique permutation that moves the order $<$ to the order $<^{\prime}$, then if $M_{\sigma}$ is the standard permutation matrix and let $\Phi=\operatorname{diag}_{v_{i}^{\prime} \in \tau^{\prime}}\left(\phi\left(v_{i}^{\prime}\right)\right)$, then $M=\Phi^{*} M_{\sigma^{-1}}$.

Of course the form of $M$ depends on the initial choice of $\phi\left(v_{0}\right)=i d$, which amounts to using the iso $U_{v_{0} v_{0}^{\prime}}^{\tau}$ to pull-back the matrix. Any other choice of iso will differ by an element of $\mathscr{A}$ which is then the value of $\phi_{v_{0}}$. This plays a crucial role later.

3.2.3. Commutative case. Reduced gauge group. In the commutative case, we can fix a character $\chi: \mathscr{A} \rightarrow \mathbb{C}$ and then under $\hat{\chi}$ all matrices become $U(1)$ valued and all the Hilbert spaces $\mathscr{H}_{v}$ become identified with $\mathbb{C}$. In this case, we can identify the gauge group action with an 
action of $U(1)^{\times V_{\Gamma}}$ on $U(1)$-valued weight functions, using $\lambda=\chi \circ \phi$. For every oriented edge $\vec{e}$ from $v$ to $w$ the re-gauged weights are

$$
w t^{\prime}(\chi(\vec{e})):=\lambda(v) \chi(w t(\vec{e})) \bar{\lambda}(w)
$$

Notice that we have taken the indexed un-ordered product. If we fix an order of the vertices, then the group $\mathbb{S}_{k}$ acts on the vertices as well and the full gauge group which acts on the Hamiltonians by conjugation is the wreath product $G=U(1) \imath \mathbb{S}_{k}$.

We see that the constant functions $\lambda$ act trivially and hence to get a more effective action we can quotient by the diagonal $U(1)$ action and consider the reduced gauge group $\bar{G}:=G / U(1)$, where $U(1)$ is diagonally embedded in $U(1)^{k}$ and $\mathbb{S}_{k}$ acts trivially.

Abstractly $U(1)^{k} / U(1) \simeq U(1)^{k-1}$, to make this explicit, we can choose a section of $\bar{G} \rightarrow G$. Our choice $\phi\left(v_{0}\right)=1$ is just such a choice of a section. The action of $\mathbb{S}_{k}$ on the remaining $k-1$ factors is then more involved, however. It is still a semi-direct product, but not a wreath product any more. This has practical relevance in the Gyroid case.

The proof of the theorem above then boils down to the fact that a rooted spanning tree uniquely fixes a unique gauge transformation as follows. We let $\lambda$ (root) $=1$ by the global gauge $U(1)$. Now the weight on each vertex of the tree is fixed iteratively by the condition that $\lambda w t(e)=1$. The whole set of weights then gives a diagonal unitary matrix and taking the product with the appropriate permutation, we obtain the matrix $M$.

3.2.4. Gauge groupoid. Let $\operatorname{Ham}(\bar{\Gamma}, \mathscr{A})$ be the set of all matrix Hamiltonian $H_{\tau,<}$ with all possible rooted spanning trees with compatible weight functions $w t: E_{\bar{\Gamma}} \rightarrow \mathscr{A}$. Let $U(\mathscr{A})$ be the unitary elements of $\mathscr{A}$. Then the full gauge group $G=U(\mathscr{A})^{k} 々 \mathbb{S}^{k}$ acts on the orders and the weight function and on the Hamiltonians by conjugation and permutation just as above.

Given a concrete Harper Hamiltonian, $H_{0}$, its set of possible matrix versions $\operatorname{Ham}_{0} \subset \operatorname{Ham}$ given by the different choices on $(\tau,<)$ are contained in the orbit of one such particular choice. In this situation, we want to restrict to a groupoid. Namely for each point $H_{\tau,<} \in \operatorname{Ham}_{0}$, we want to only consider those elements in $G$ which send $H_{\tau,<}$ to another element in $H_{a m}$. By what we have discussed so far, for each two elements in this orbit, there is an element in $G$ which takes one element to the other and we did give a particular choice for it. These elements constitute a groupoid or rather give a representation of a groupoid $\mathcal{G}$. Abstractly, $\mathcal{G}$ has objects pairs $(\tau,<)$ of a rooted spanning tree 
and an order of $\bar{\Gamma}$ and a unique isomorphism between any two such pairs. If the two pairs coincide, the isomorphism is the identity map. The representation is given by mapping $(\tau,<)$ to $H_{\tau,<}$ and the unique isomorphism to the morphism of Theorem 3.3.

3.2.5. Matrix version and cocycle. In order to calculate it is preferable to work with a matrix representation of the groupoid action. The problem is that although the groupoid associates a matrix to each regauging, these matrices all act in different spaces. To make everything coherent, we have to fix once and for all a base vertex $v_{*}$ and isomorphisms $U_{v v_{*}}: \mathscr{H}_{v_{*}} \rightarrow \mathscr{H}_{v}$. We do this by using some initial choice of spanning tree $\left(\tau_{*}, v_{*},<_{*}\right)$ and set $U_{v v_{*}}:=U_{v v_{*}}^{\tau_{*}}$.

Now, if we are given three choices $H_{\tau_{0},<_{0}}, H_{\tau^{\prime},<^{\prime}}$ and $H_{\tau^{\prime \prime},<^{\prime \prime}}$, let $g_{1}=$ $M U_{v_{*} v_{0}}^{\tau}$ be the morphism of Theorem 3.3 for moving from $H_{\tau,<}$ to $H_{\tau^{\prime},<^{\prime}}$ by conjugation, and likewise define $g_{2}, M_{2}$ for the move from $H_{\tau^{\prime},<^{\prime}}$ to $H_{\tau^{\prime \prime},<^{\prime \prime}}$ and $g_{3}, M_{3}$ the morphism and matrix associated to the move from $H_{\tau,<}$ to $H_{\tau^{\prime \prime},<\prime \prime}$.

Then as morphisms $g_{1} g_{2}=g_{3}$ as one can check by direct calculation. If we naively multiply the matrices $M_{1}$ and $M_{2}$ and think of the product as an endomorphism of $\bigoplus_{i} \mathscr{H}_{v_{*}}$, then we are tacitly using a pull-back of the endomorphism given $M_{1}$ on $\bigoplus_{i} \mathscr{H}_{v_{0}}$. For $M_{2}$ because of the definition of $M$, we first pull-back from $\bigoplus_{i} \mathscr{H}_{v_{0}^{\prime}}$ to $\bigoplus_{i} \mathscr{H}_{v_{0}}$ using $\tau$ and then further to $\bigoplus_{i} \mathscr{H}_{v_{*}}$, that is the matrix product $M_{1} M_{2}$ represents the morphisms $\tilde{M}_{1} \circ \tilde{M}_{2}$ where $\tilde{M}_{1}=U_{v_{*} v_{0}^{\prime}}^{\tau_{*}} M_{1} U_{v_{0} v_{*}}^{\tau_{*}}, \tilde{M}_{2}=$ $U_{v_{*} v_{0}}^{\tau_{*}} U_{v_{0} v_{0}^{\prime}}^{\tau} M_{2} U_{v_{0}^{\prime} v_{0}}^{\tau} U_{v_{0} v_{*}}^{\tau_{*}}$. Now, we get the matrix equation $\tilde{M}_{1} \circ \tilde{M}_{2}=$ $\widetilde{C \circ M_{3}}$ with $C=U_{v_{0} v_{0}^{\prime}}^{\tau} U_{v_{0}^{\prime} v_{0}^{\prime \prime}}^{\tau^{\prime}} U_{v_{0}^{\prime \prime} v_{0}}^{\tau}$. Now $C$ need not be the identity in general, but it is some element in $U(\mathscr{A})$. More precisely, we have $U_{v_{0} v_{0}^{\prime}}^{\tau} U_{v_{0}^{\prime} v_{0}^{\prime \prime}}^{\tau^{\prime}} U_{v_{0}^{\prime \prime} v_{0}}^{\tau} \in \mathscr{A}$ is a diagonal/scalar matrix. In the case of a wire network we have that $\mathscr{A}=\mathbb{T}_{\Theta}^{n}$ and the matrix above is just of the form $\alpha U_{\vec{e}}$ with $\vec{e} \in L$.

Summing up, we consider the map $\rho: \mathcal{G} \rightarrow G L_{k}(\mathscr{A})$ given by associating to the groupoid element $g$ moving from $H_{\tau^{\prime},<^{\prime}}$ and $H_{\tau^{\prime \prime},<^{\prime \prime}}$ the matrix $M$ constructed in Theorem 3.3. This map is not in general a groupoid representation by itself. As we just calculated for two composable matrices $g_{1}, g_{2}$ let $c\left(g_{1}, g_{2}\right)$ be the matrix representing the morphism $C$ then

$$
\rho\left(g_{1}\right) \rho\left(g_{2}\right)=c\left(g_{1}, g_{2}\right) \rho\left(g_{1} g_{2}\right)
$$

Using the definitions one calculates that

$$
c\left(g_{1}, g_{2}\right) c\left(g_{1} g_{2}, g_{3}\right)=c\left(g_{1}, g_{2} g_{3}\right) c\left(g_{2}, g_{3}\right)
$$


the value being

$c\left(g_{2}, g_{2}, g_{3}\right)$ which in the analogous notation is given by $U_{v_{0} v_{0}^{\prime}}^{\tau} U_{v_{0}^{\prime} v_{0}^{\prime \prime}}^{\tau^{\prime}} U_{v_{0}^{\prime \prime} v_{0}^{\prime \prime \prime}}^{\tau^{\prime \prime}} U_{v_{0}^{\prime \prime \prime} v_{0}}^{\tau}$. So technically, we have a non-commutative groupoid 2-cocycle with values in $\mathbb{T}_{\Theta}^{n}$ which is given by $c(g, h):=M_{g} M_{h} M_{g h}^{-1}$ which is defined for any two composable re-gaugings (see [8] for a definition).

One good way, albeit a bit abstract, to view the matrix multiplication, is that we are dealing not with a representation of the groupoid per $s e$, but rather, with representations of $\pi_{1}$ of the re-gauging groupoid and our initial choice of $\left(\tau_{*}, v_{*},<_{*}\right)$ is the choice of a base point. The correspondence is analogous to the one discussed in $\S 2.2 .1$.

3.2.6. Re-gaugings Induced by Graph Symmetries. If we have a symmetry, aka. automorphism, of the graph $\bar{\Gamma}$, then given a fixed choice $(\tau,<)$, we can push forward both these pieces of data to $\left(\tau^{\prime},<^{\prime}\right)$ with $\phi$. This means that for every $H_{\tau,<}$ any automorphism $\phi$ gives rise to a re-gauging of $H_{\tau,<}$ to $H_{\tau^{\prime},<^{\prime}}$.

3.2.7. Lifts to Automorphisms. One interesting question for any given re-gauging is if there are automorphisms $\psi$ of $\mathscr{A}$ such that

$$
\hat{\psi}\left(H_{\tau,<}\right)=U_{v_{0} v_{0}^{\prime}}^{\tau} H_{\tau^{\prime},<^{\prime}} U_{v_{0}^{\prime} v_{0}}^{\tau}
$$

where, again, $\hat{\psi}$ is $\psi$ applied to the entries. This is the type of enhanced, extended symmetry we will use in the commutative case.

One way such a symmetry can arise is by a re-gauging induced by an automorphism $\phi$ is $\bar{\Gamma}$. A stricter requirement that is easier to handle is that not only the matrix coefficients of the Hamiltonian transform into each other, but rather already the weight functions. This avoids dealing with sums of weights. We say a re-gauging induced by an automorphism $\phi$ of $\bar{\Gamma}$ is weight liftable by an automorphism $\psi$ of $\mathscr{A}$ if $\psi(w t(\vec{e}))=w t^{\prime}(\phi(\vec{e}))$, where $w t^{\prime}$ is the re-gauged weight function for the pushed forward spanning tree.

Theorem 3.5. Given an automorphism $\phi$ of $\bar{\Gamma}$ there is at most one weight lift by an automorphism $\psi$ of the re-gauging induced by $\phi$. On the generators $w t(\vec{e})$, e not a spanning tree edge, the putative map is fixed by the condition $\psi(w t(\vec{e})):=w t^{\prime}(\phi(\vec{e}))$, where $w t^{\prime}$ is the regauged weight function.

Furthermore, the $\psi(w t(\vec{e}))$ again generate $\mathscr{A}$ and hence whether $\psi$ indeed defines an automorphism only needs to be checked on the generators $w t(\vec{e})$.

Lastly, $\psi$ is induced by a base change of $\pi_{1}(\bar{\Gamma})$. 
Proof. Let $w t^{\prime}$ be the re-gauged weights after moving from $(\tau,<)$ to $\left(\tau^{\prime},<^{\prime}\right)$. If an automorphism $\psi$ of $\mathscr{A}$ that lifts $\phi$ exists, then it satisfies $w t^{\prime}(\phi(\vec{e}))=\psi(w t(\vec{e}))$. After fixing an orientation for each edge the $w t(\vec{e})$ generate, we see that the morphism is already fixed, since by assumptions the $w t(\vec{e})$ generate $\mathscr{A}$.

In order to show that the $\psi(w t(\vec{e}))$ are generators, we will prove the last statement first. As discussed in $\S 2.2 .1 w t$ gives a representation $\rho$ of $\pi_{1}\left(\bar{\Gamma}, v_{0}\right)$ and $w t^{\prime}$ gives a representation $\rho^{\prime}$ of $\pi_{1}\left(\bar{\Gamma}, v_{0}^{\prime}\right)$ if $v_{0}$ is the root of $\tau$ and $v_{0}^{\prime}=\phi\left(v_{0}\right)$ is the root of $\tau^{\prime}$, the pushed forward spanning tree. In $\tau$ there is a canonical shortest path $p_{v_{0} v_{0}^{\prime}}^{\tau}$ from $v_{0}^{\prime}$ to $v_{0}$. Conjugating by this path gives an isomorphism $P: \pi_{1}\left(\bar{\Gamma}, v_{0}^{\prime}\right) \rightarrow \pi_{1}\left(\bar{\Gamma}, v_{0}\right)$. This is in essence the definition of the path groupoid of $\bar{\Gamma}$. Let $l^{\tau}(\vec{e})$ be the loop associated to $\vec{e}$ by using $\tau$ as a spanning tree, see $\S 2.2 .1$, then $w t(\vec{e})=\rho\left(l^{\tau}(\vec{e})\right)$. It follows from the definition of the re-gauging that $\psi\left(\rho\left(l^{\tau}(\vec{e})\right)\right)=\psi(w t(\vec{e}))=w t^{\prime}(\phi(\vec{e}))=\rho^{\prime}\left(l^{\tau^{\prime}}(\phi(\vec{e}))\right)=\rho\left(P\left(l^{\tau^{\prime}}(\phi(\vec{e}))\right)\right)$ so that $\psi$ is induced by the chance of basis $l^{\tau}(\vec{e}) \rightarrow P\left(l^{\tau^{\prime}}(\phi(\vec{e}))\right)$ in $\pi_{1}\left(\bar{\Gamma}, v_{0}\right)$. From this it follows that the $\psi(w t(\vec{e}))$ generate.

Corollary 3.6. If the groupoid representation is non-degenerate, so that $\mathscr{A}$ is generated by the wt $(\vec{e})$ and each non-spanning-tree edge gives a linearly independent generator, then the morphism $\Psi$ above is well-defined as a linear morphism.

If there are no relations among the generators, e.g. in the case $\mathscr{A}=$ $\mathbb{T}^{n}$ the commutative algebra of the torus, then every automorphism is weight liftable, i.e. $\Psi$ from above is well-defined as an algebra homomorphism.

We will use the corollary in $\S 3.3$ to define the enhanced symmetry groups in the commutative toric non-degenerate case.

Remark 3.7. These types of symmetries might also help to explain the somewhat mysterious approximate symmetry between the noncommutative and the commutative case found in [6]. Here the symmetry is between two loci in $B$. Where $B$ in the commutative case is the base for the family of Hamiltonians as discussed above and in the noncommutative case is the space parameterizing the background magnetic field. The two loci are the locus of degenerate Eigenvalues on the commutative side and the locus of values of $B$ where $\mathscr{B}$ is not the full matrix algebra. From the examples PDG and honeycomb, these two loci have exactly the same top dimension and there are further characteristic features which they have in common. These considerations would lead 
us too far astray in the present context, but we plan to return to them in a future paper.

3.3. Enhanced symmetries in the commutative case. We will concentrate on the commutative case in the following. One physical feature that makes the non-commutative theory more complicated is that conjugating $H$ with elements from $\mathscr{A}$ usually does not leave it invariant. This is of course the starting point for considering the $C^{*}-$ algebra $\mathscr{B}$ which contains all these conjugates.

3.3.1. Extension. In the commutative case, $U(\mathscr{A})$ is a commutative group and the 2-cocycle defines a central extension $\tilde{\mathcal{G}}$ of $\mathcal{G}$ by $U(\mathscr{A})$. We can consider the action of this central extension, since the action of $U(\mathscr{A})$ commutes with the Hamiltonians, permutations and the regaugings in this case

$$
1 \rightarrow U(\mathscr{A}) \rightarrow \tilde{\mathcal{G}} \rightarrow \mathcal{G} \rightarrow 1
$$

Then $\rho$ does give a groupoid representation of $\tilde{\mathcal{G}}$.

Remark 3.8. There is a nice geometric interpretation of this in the case of wire networks. Here the group $U(\mathscr{A})$ corresponds to translations along the lattice $L$. One can identify the vertices of $\bar{\Gamma}$ with the elements in chosen primitive cell and likewise one can arrange the spanning tree edges to be inside this cell. When we are re-gauging, we move the base point along the spanning tree edges. After doing this several times the new root can lie outside the original primitive cell. The co-cycle then measures the displacement of the new cell relative to the old cell in terms of an element $\lambda \in L$, more precisely it is just $U_{\lambda}$.

3.3.2. Enhanced symmetry Group. In order to find degeneracies in the spectrum, we use the characters and then look for fixed points under the induced groupoid action. Using the language of $\S 2.2 .4$, given a point $\chi \in \mathscr{A}$ we get a map $\hat{\chi}: \operatorname{Ham}_{0} \rightarrow M_{k}(\mathbb{C})$. There is then an induced action of the groupoid on $\hat{\chi}\left(\operatorname{Ham}_{0}\right)$, by pushing forward with this character. It can now happen that $\hat{\chi}\left(H_{\tau,<}\right)=\hat{\chi}\left(H_{\tau^{\prime},<^{\prime}}\right)$, that is $H_{\tau,<}(t)=H_{\tau^{\prime},<^{\prime}}(t)$, for the point $t \in B$ corresponding to $\chi$.

For each element $H(t) \in \hat{\chi}\left(\operatorname{Ham}_{0}\right)$, we get its stabilizer group $S t(H(t))$ under the induced groupoid action. This is the image of the transitive action of the groupoid on the fiber of $\hat{\chi}$ over $H(t)$. We can identify $S t(H(t))$ with the image of that subgroupoid. If this group is not trivial, which means that the fiber is not just a point, we call this group the enhanced symmetry group of $H(t)$. It is realized by re-gaugings, that is 
conjugation by specific matrices which form a projective representation of the stabilizer group as we presently discuss.

3.3.3. Super-selection rules, Projective Representation and Degeneracies. If $S t(H) \neq 1$ then this means that the set of all matrices $\hat{\chi} \rho(g)$ for $g \in S t(H(t))$, where we identified $g$ with its defining element in $\mathcal{G}$, all commute with the Hamiltonian $H(t)$ and hence each one and all of them together give super-selection rules. This of course is already a great help in finding the spectrum.

Since $\rho$ is only a groupoid representation of $\tilde{\mathcal{G}}$, we get that $\hat{\chi} \rho$ is a representation of a an extension of $S t(H(t))$. Since we are in the commutative case, this extension is central and gives rise to a projective representation of $S t(H(t))$.

$$
1 \rightarrow U(1) \rightarrow \tilde{S} t(H(t)) \rightarrow S t(H(t)) \rightarrow 1
$$

In order to apply the general arguments of representation theory, we will be interested in the class of this extension. These extensions are classified up to isomorphism by $H^{2}(S t(H(t)), U(1))[9,10]$.

We give a brief definition of this cohomology group, as it is important for our calculations (see e.g. [11]). Let $G$ be a group and $A$ be an Abelian group, which we also write multiplicatively ${ }^{2}$. Set $C^{i}(G, A):=$ $\operatorname{Map}\left(G^{\times i}, U(1)\right)$ these are the $i$-th cochains. There is a general differential $d: C^{i} \rightarrow C^{i+1}$ with $d^{2}=0$. We will need the formulas for it on 1and 2- cochains. If $\lambda \in C^{1}(G, A)$ then $d \lambda(g, h)=\lambda(g) \lambda(h) \lambda(g h)^{-1}$ and if $c \in C^{2}(G, A)$ then $d c(g, h, k)=c(h, k) c^{-1}(g h, k) c(g, h k) c(g, h)^{-1}$. Set $Z^{2}(G, A):=\operatorname{ker}\left(d: C^{2}(G, A) \rightarrow C^{3}(G, A)\right)$ and $B^{2}(G, A):=\operatorname{Im}(d:$ $\left.C^{1}(G, A) \rightarrow C^{2}(G, A)\right)$. Notice that an element $c$ is $Z^{2}$ precisely means that $c$ satisfies the cocycle condition (8). Now $B^{2}(G, A) \subset Z^{2}(G, A)$ and $H^{2}(G, A):=Z^{2}(G, A) / B^{2}(G, A)$.

What this means is that we can move to an isomorphic extension by using a rescaling $\lambda \in C^{1}(S t(H(t)), U(1))$. Another interesting concrete question is if a given homology class $[c]$ can be represented by a cocycle in a subgroup of $A$. This is especially interesting if the subgroup is finite. In our concrete calculations for the Gyroid, we will use for instance $\mathbb{Z} / 2 \mathbb{Z}$ and this will lead us to consider double covers.

In general if we identify that the projective action of $S t(H(t))$ is isomorphic to an action of a finite group extension $\tilde{S} t(H(t))$, then we can use this representation to decompose $\mathbb{C}^{k}$ into its isotypical decompositions with respect to this group action. If the group is non-Abelian, then there is a chance that some of the irreducible representations in

\footnotetext{
${ }^{2}$ We consider $A$ to be a $G$ module with the trivial action.
} 
the decomposition are higher dimensional, which implies degeneracies of the order of these dimensions. Again this is present for the Gyroid.

3.3.4. Geometric lift of the groupoid action. In order to understand the (projective) group action, geometrically in the commutative case, one lifts the action on the Hamiltonians to an action on the underlying geometric space. We will now for concreteness fix $\mathscr{A}=\mathbb{T}^{n}$, that is the groupoid representation is commutative, toric non-degenerate, as is the case in all crystal examples we consider: PDG, Bravais and Honeycomb.

Finding lifts then means that one considers the commutative diagram

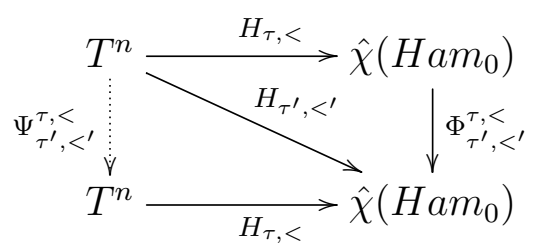

where the dotted morphism is the lift to be constructed and $H_{\tau,<}$ is the map $t \mapsto H_{\tau,<}(t):=\hat{\chi}(t)$ if $\chi$ is the character corresponding to $t$.

The existence of these lifts is not guaranteed in general, and indeed there are examples of re-gaugings that cannot be lifted. A non-liftable example can e.g. be produced from the cube graph obtained from the Gyroid graph by quotienting out by the the simple cubic lattice, see [2]. We will show that all lifts stemming from automorphisms of the underlying graph do lift.

Looking at the diagram (12), one consequence of this action is that it lets us pinpoint Hamiltonians with enhanced symmetry group. Using Corollary 3.6 and translating it to the geometric side, we obtain

Proposition 3.9. Let $(\bar{\Gamma}, w t)$ be a toric non-degenerate weighted graph. In the commutative case the automorphism group of $\bar{\Gamma}$ lifts via the gauging action to an automorphism group of $\mathbb{T}^{n}$. That is we get a morphism $\operatorname{Aut}(\bar{\Gamma}) \rightarrow \operatorname{Aut}\left(\mathbb{T}^{n}\right)$.

If a point $t \in T^{n}$ is a fixed point of a lift of an element $g \in \mathcal{G}$, then, the re-gauging is an enhanced symmetry for the corresponding Hamiltonian, that is $\hat{\chi}(\rho(g))$ commutes with the Hamiltonian $H_{\tau,<}(t)$.

Summarizing these results:

Theorem 3.10. If $(\bar{\Gamma}, w t)$ is commutative and toric non-degenerate, then a stabilizer sub-group $G_{t}$ of $t \in \mathbb{T}^{n}$ under the induced action of Aut $(\bar{\Gamma})$ on $T^{n}$ leads to an enhanced symmetry group $S t(H(t))$ for the 
24 RALPH M. KAUFMANN, SERGEI KHLEBNIKOV, AND BIRGIT KAUFMANN

Hamiltonian $H(t)$. This group also has a projective representation via the matrices $\hat{\chi}(\rho(g))$.

We can exploit the representation theory of this group to get information about degeneracies.

Example 3.11. For commutative toric non-degenerate groupoid representations of symmetric graphs the re-gaugings by re-orderings are always representable via an automorphism of the graph. If $\sigma \in \mathbb{S}_{k-1}$ permutes the vertices of the spanning tree leaving the root fixed, then the re-gauging lifts as the reordering of the generators and possibly taking $*$ of them. The matrices are just the usual permutation matrices of $\mathbb{S}_{k-1} \subset \mathbb{S}_{k}$ acting on the last $k-1$ copies of $\mathbb{C}$ in $\mathbb{C}^{k}$.

Remark 3.12. In the commutative case, the representation of $\pi_{1}\left(\bar{\Gamma}, v_{0}\right)$ factors through its Abelianization $H_{1}(\bar{\Gamma})$.

\section{Calculations and Results for Wire Networks}

In this paragraph, we perform the calculation for the general theory we developed for the PDG and honeycomb wire networks as described in 2.1. In all these situations Theorem 3.10 applies. The upshot of the following calculations together with the analysis of [3] is:

Theorem 4.1. In all the examples $P D G$ and honeycomb, all the fixed points come from fixed points of the semi-classical action. Moreover the fixed points, stabilizer groups, their extensions and the decomposition into irreps for the case of the Gyroid are given in Table 2.

4.1. Gyroid. The graph $\bar{\Gamma}$ in the Gyroid case is the full square. It has symmetry group $\mathbb{S}_{4}$. With the Gyroid weights, the graph is faithful and hence the action can be lifted to an action on the torus. It acts transitively on all ordered spanning trees. Such a spanning tree is fixed by specifying a root and the order. The subgroup of $\mathbb{S}_{3}$ acts transitively on all orders. The matrices of this subgroup action are just the permutation matrices acting on the last 3 copies of $\mathbb{C}$ in $\mathbb{C}^{4}$ and the lift of the $\mathbb{S}_{3}$ action on the generators $A, B, C$ of $\mathbb{T}^{3}$ is given by the permutation action.

We fix an initial rooted spanning tree and order as in Figure (3).

4.1.1. Action on $T^{n}$. The action of $\mathbb{S}_{4}$ on $T^{3}$ is fixed once we know the action of the generators (12), (23) and (34).

The action of (23) is graphically calculated in Figure 2, from which one reads off $\Psi((23))(A, B, C)=\left(A^{*}, C^{*}, B^{*}\right)$. Here $(A, B, C)$ is the notation for the initially chosen basis of $\mathbb{T}^{3}$. 

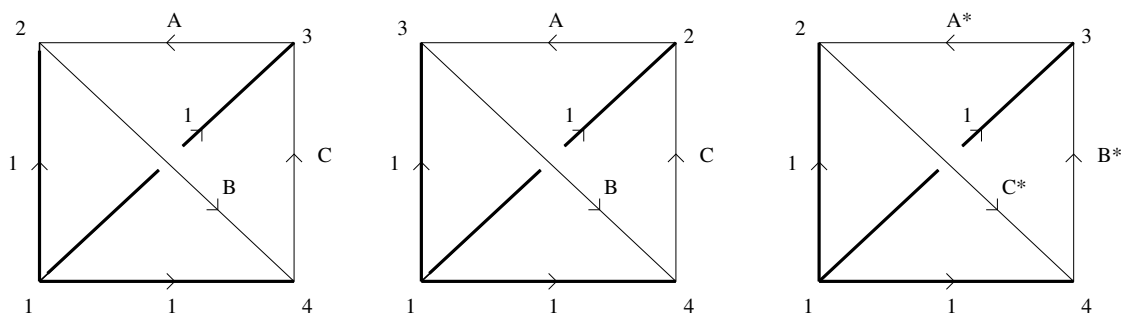

Figure 2. Calculation of the action of $(23)$ on $T^{3}$. The original graph, the pushed forward order and the move into the old position to read off the morphism

In the graphical calculation, we first write down the graph together with the initial spanning tree and order. We then push-forward the spanning tree and the order. For this we keep the vertices and edges as well as the weights fixed. We then (if necessary) give the re-gauging parameters by writing them next to the respective vertices and (if necessary) perform the re-gauging. Finally we move the vertices and edges, so that they coincide with their pre-images to read off the morphism on the generators given by Theorems 3.5 and 3.10 .

A similar calculation shows that $\Psi((34))(A, B, C)=\left(B^{*}, A^{*}, C^{*}\right)$. A consequence is that the cycle $(234)=(23)(34)$ acts as $\Psi((234))(A, B, C)=$ $\Psi((23))\left(B^{*}, A^{*}, C^{*}\right)=(B, C, A)$ and is the cyclic permutation.

The action of (12) is more complicated as the root is moved. For this we calculate graphically, see Figure 3 , and read off $\Psi$ as: $(A, B, C) \mapsto$ $\left(A^{*}, B^{*}, A C B\right)$.

This allows us to compute fixed points and stabilizer groups. We will first concentrate on non-Abelian stabilizer groups. There are only two fixed points under the full $\mathbb{S}_{4}$ action and these are $(1,1,1)$ and $(-1,-1,-1)$. The group $A_{4}$, the subgroup of all even permutations, is the stabilizer group of the two points $(i, i, i)$ and $(-i,-i,-i)$. One can readily check that these are the only non-Abelian stabilizer groups. The other possibility would be $\mathbb{S}_{3}$, but a short calculation shows that anything that is stabilized by any $\mathbb{S}_{3}$ subgroup is stabilized by all of $\mathbb{S}_{4}$.

4.1.2. Representations. We collect together the matrices $M$ needed for further calculation. Again, we fix our initial ordered rooted spanning tree as before.

Using short hand notation, the matrix for the re-gauging induced by the transpositions $(12),(13),(14)$ from the initial spanning tree to 

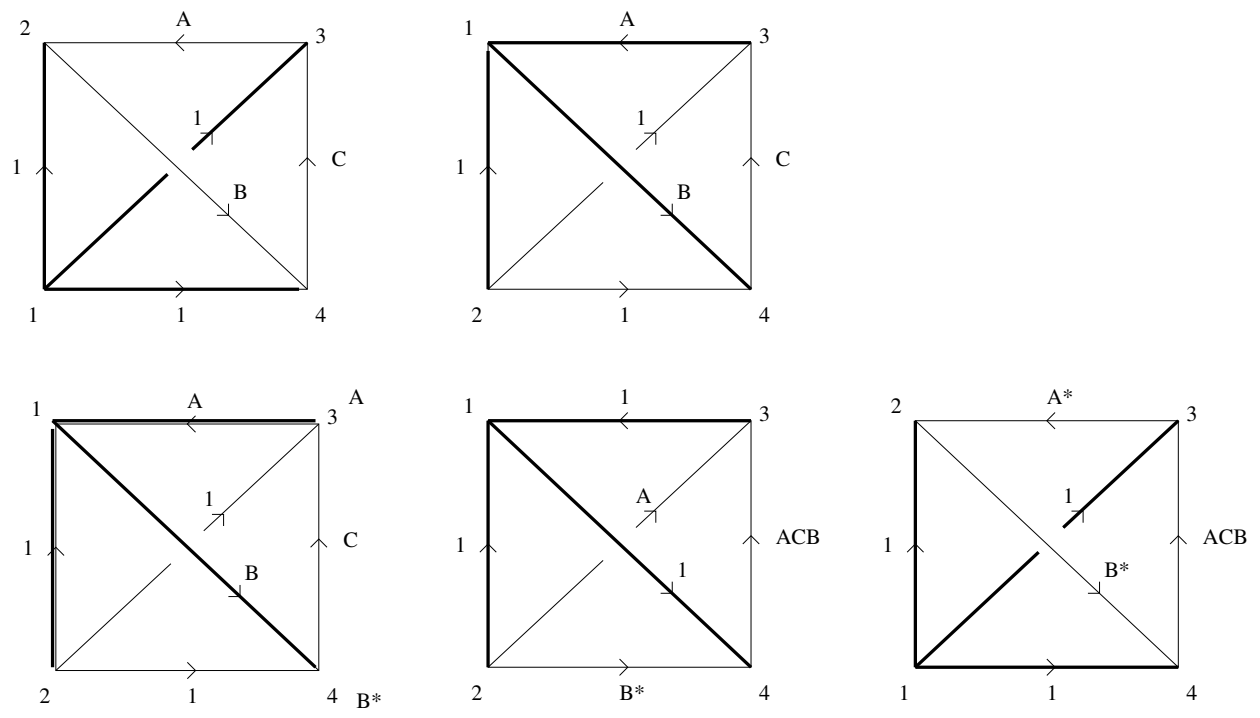

Figure 3. Calculation of the action of (12) on $T^{3}$

the pushed forward one are

$\rho_{12}=\left(\begin{array}{cccc}0 & 1 & & \\ 1 & 0 & & \\ & & A & \\ & & & B^{*}\end{array}\right), \rho_{13}=\left(\begin{array}{cccc}0 & & 1 & \\ & A^{*} & & \\ 1 & & 0 & \\ & & & C\end{array}\right), \rho_{14}=\left(\begin{array}{cccc}0 & & & 1 \\ & B & 0 & \\ & 0 & C^{*} & \\ 1 & & & 0\end{array}\right)$

The calculation for $\rho_{12}$ can be read off from Figure 3. For this we read off the matrix $\Phi$ from the re-gauging parameter and the matrix $M_{\sigma}$ is given by the permutation we are considering. The other calculations are similar. All other transpositions, viz. those not involving 1, simply yield permutation matrices as there is no re-gauging involved. It is convenient to also have the following matrices as a reference:

$$
\rho_{(12)(34)}=\left(\begin{array}{cccc}
0 & 1 & & \\
1 & 0 & & \\
& & 0 & A \\
& & B^{*} & 0
\end{array}\right), \quad \rho_{(14)(23)}=\left(\begin{array}{ccc} 
& & \\
& C^{*} & \\
1 & &
\end{array}\right)
$$

and finally

$$
\rho_{(123)}=\left(\begin{array}{cccc}
0 & 0 & 1 & \\
1 & 0 & 0 & \\
0 & A & 0 & \\
& & & B^{*}
\end{array}\right)
$$

4.1.3. The point $(0,0,0)$. At $(0,0,0)$, the matrices $\rho_{12}, \rho_{23}, \rho_{34}$ give the usual representation of $\mathbb{S}_{4}$ on $\mathbb{C}^{4}$. As is well known this representation 
decomposes into the trivial representation and an irreducible 3-dim representation. This means that there is an at least 3 -fold degenerate Eigenvalue $\lambda$. Since the trace of $H$ is zero, we also know that the Eigenvalues satisfy $\mu=-3 \lambda$. Plugging in $(1,1,1,1)$, which spans the trivial representation, we see that $\mu=3$ and $\lambda=-1$.

4.1.4. The point $(\pi, \pi, \pi)$. In this case, the matrices $\rho_{12}, \rho_{23}, \rho_{34}$ only give a projective representation. As one can check that $\rho_{12} \rho_{23} \rho_{12}=$ $-\rho_{13}$ while $\rho_{23} \rho_{12} \rho_{23}=\rho_{13}$ for instance. Define the 1 -cocycle $\lambda$ by $\lambda(\sigma)=(-1)$ if 1 appears in a cycle of length $>1$ and 1 else. So that $\lambda((12))=\lambda((13))=\lambda((123))=-1$ while $\lambda((23))=\lambda((24))=$ $\lambda((234))=1$. Then one calculates that $\tilde{\rho}:=\rho \circ \lambda$ has a trivial cocycle $c$ and thus $\rho$ is isomorphic to a true linear representation of $\mathbb{S}_{4}$. Checking the characters, one sees again that in this case the irreducible components of $\tilde{\rho}$, which also commute with $H$ is again the one-dimensional trivial representation and the 3-dimensional standard representation. The trivial representation is spanned by $(-1,1,1,1)$. The Eigenvalues are then readily computed to be 1 with multiplicity 3 and -3 with multiplicity 1.

Remark 4.2. We would like to remark that the choice of $\lambda$ amounts to choosing a different gauge for the root vertex, namely -1 instead of 1.

Remark 4.3. Notice that already in this case, even though there is no projective extension, our enhanced gauge-group is necessary. Without it there would only be an $\mathbb{S}_{3}$ action, those elements which involve no re-gauging. This smaller symmetry group is, however, not powerful enough to force the triple degeneracy, as $\mathbb{S}_{3}$ has no irreducible 3-dim representation.

4.1.5. The point $(\pi / 2, \pi / 2, \pi / 2)$ and $(-\pi / 2,-\pi / 2,-\pi / 2)$. These points are similar to each other. We will treat the first one in detail. Again, we have only a projective representation of $A_{4}$ aka. the tetrahedral group $T$. Namely, $\rho_{(12)(23)} \rho_{(13)(24)}=-i \rho_{(14)(23)}$. Again we can scale by a 1 -cocycle $\lambda$. This time $\lambda(i d)=1, \lambda((i j)(k l))=i, \lambda(i j k)=1$ if $1 \notin\{i, j, k\}$, and $\lambda((i j k))=i$ if $1 \in\{i, j, k\}$. The resulting representation $\tilde{\rho}=\rho \circ \lambda$ is then still a projective representation, but is it a representation of the unique non-trivial $\mathbb{Z} / 2 \mathbb{Z}$ extension of $A_{4}$, which goes by the names $2 T, 2 A_{4}, S L(2,3)$ or the binary tetrahedral group. This group is well known. It is presented by generators $s$ and $t$ with the relations $s^{3}=t^{3}=(s t)^{2}$. In $S L(2,3)$ (that is the special linear 
group of $2 \times 2$ matrices over the field with three elements $\mathbb{F}_{3}$ ), one can choose $s=\left(\begin{array}{cc}-1 & -1 \\ 0 & -1\end{array}\right)$ and $t=\left(\begin{array}{cc}-1 & 0 \\ -1 & -1\end{array}\right)$.

For $2 A_{4}$ using a set theoretic section $\wedge$ of the extension sequence

$$
1 \longrightarrow \mathbb{Z} / 2 \mathbb{Z} \longrightarrow 2 A_{4} \underset{\wedge}{\longrightarrow} A_{4} \longrightarrow 1
$$

and $z$ as a generator for $\mathbb{Z} / 2 \mathbb{Z}$, we can pick $s=z \widehat{(123)}, t=z \widehat{(234)}$ as generators. Now we can check the character table, Table 1 , and find that the representation $\tilde{\rho}$ over the complex numbers decomposes as the sum of two irreducible two-dimensional representations $\chi_{5} \oplus \chi_{6}$. In fact, these are the two representations into which the unique real irreducible 4 -dimensional representation of complex type splits over $\mathbb{C}$.

The explicit computation for the representation

$$
\begin{aligned}
& \tilde{\rho}(s)=-\lambda((123)) \rho_{(123)}=\left(\begin{array}{cccc}
0 & 0 & -i & 0 \\
-i & 0 & 0 & 0 \\
0 & 1 & 0 & 0 \\
0 & 0 & 0 & -1
\end{array}\right) \\
& \tilde{\rho}(t)=-\lambda((234)) \rho_{(234)}=-\left(\begin{array}{cccc}
1 & 0 & 0 & 0 \\
0 & 0 & 0 & 1 \\
0 & 1 & 0 & 0 \\
0 & 0 & 1 & 0
\end{array}\right)
\end{aligned}
$$

is as follows. Suppose the $\tilde{\rho}=\bigoplus_{i=1}^{7} a_{i} \rho_{i}$, where $\rho_{i}$ is the irrep with character $\chi_{i}$. Now $\operatorname{tr}(i d)=4, \operatorname{tr}(-1)=-4$, using the character table this implies that the coefficients $a_{1}=a_{2}=a_{3}=a_{7}=0$ and furthermore (*) $a_{4}+a_{5}+a_{6}=2$. We furthermore have that $\operatorname{tr}(s)=-1$ so that $a_{4}+\omega a_{5}+\omega^{2} a_{6}=-1$ which together with $\left(^{*}\right)$ implies that $a_{4}=0, a_{5}=$ $a_{6}=1$. This fixes the decomposition into irreps. As a double check one can verify that the rest of the equations are also satisfied.

So indeed we find that $(\pi / 2, \pi / 2, \pi / 2)$ is a point with two Eigenvalues with degeneracy 2. It is not hard to find (e.g. using the results of $\S 4.1 .6$ ) that these Eigenvalues are $\pm \sqrt{3}$.

The analysis of the complex conjugate point $(-\pi / 2,-\pi / 2,-\pi / 2)$ is analogous.

We would briefly like to connect these results to [3]. There it was shown that these four points are the only singular points in the spectrum and that the two double crossing points are Dirac points.

4.1.6. Super-Selection Rules and Spectrum Along the Diagonal. To illustrate the power of the super-selection rules we consider the action of the cyclic group generated by (234). One can easily see that the fixed 


\begin{tabular}{|c|c|c|c|c|c|c|c|}
\hline Representative & 1 & -1 & $S^{3}$ & $T^{2}$ & $S^{2}$ & $T$ & $S$ \\
\hline Elts in Conj. Class & 1 & 1 & 6 & 4 & 4 & 4 & 4 \\
\hline Order & 1 & 2 & 3 & 3 & 4 & 4 & 6 \\
\hline \hline$\chi_{1}$ & 1 & 1 & 1 & 1 & 1 & 1 & 1 \\
\hline$\chi_{2}$ & 1 & 1 & 1 & $\omega$ & $\omega^{2}$ & $\omega^{2}$ & $\omega$ \\
\hline$\chi_{3}$ & 1 & 1 & 1 & $\omega^{2}$ & $\omega$ & $\omega$ & $\omega^{2}$ \\
\hline$\chi_{4}$ & 2 & -2 & 0 & -1 & -1 & 1 & 1 \\
\hline$\chi_{5}$ & 2 & -2 & 0 & $-\omega$ & $-\omega^{2}$ & $\omega^{2}$ & $\omega$ \\
\hline$\chi_{6}$ & 2 & -2 & 0 & $-\omega^{2}$ & $-\omega$ & $\omega$ & $\omega^{2}$ \\
\hline$\chi_{7}$ & 3 & 3 & -1 & 0 & 0 & 0 & 0 \\
\hline
\end{tabular}

TABLE 1. Character table of $2 \cdot A_{4}[12]$, where $\omega=e^{\frac{2 \pi i}{3}}$.

\begin{tabular}{llllll}
$a, b, c$ & Group & $\begin{array}{l}\text { Iso class of } \\
\text { of extension }\end{array}$ & type & $\begin{array}{l}\text { Dim of } \\
\text { Irreps }\end{array}$ & Eigenvalues $\lambda$ \\
\hline$(0,0,0)$ & $\mathbb{S}_{4}$ & $\mathbb{S}_{4}$ & trivial & 1,3 & $\begin{array}{l}\lambda=-1 \text { three times } \\
\lambda=3 \text { once }\end{array}$ \\
\hline$(\pi, \pi, \pi)$ & $\mathbb{S}_{4}$ & $\mathbb{S}_{4}$ & $\begin{array}{c}\text { trivializable } \\
\text { cocycle }\end{array}$ & $\begin{array}{l}\lambda=1 \text { three times } \\
\lambda=-3 \text { once }\end{array}$ \\
\hline$\left(\frac{\pi}{2}, \frac{\pi}{2}, \frac{\pi}{2}\right)$ & $A_{4}$ & $2 A_{4}$ & $\begin{array}{l}\text { isomorphic } \\
\text { extension }\end{array}$ & 2,2 & $\lambda= \pm \sqrt{3}$ twice each \\
\hline$\left.\frac{3 \pi}{2}, \frac{3 \pi}{2}, \frac{3 \pi}{2}\right)$
\end{tabular}

TABLE 2. Possible choices of parameters $(a, b, c)$ leading to non-Abelian enhanced symmetry groups and degenerate eigenvalues of $H$

point set in the $T^{3}$ is the diagonal $t=(a, a, a)$. The matrices $\hat{\chi} \rho$ actually give a bona fide representation of $\mathbb{C}^{4}$. This is the representation of $C_{3}$ given by cyclicly permuting the last three factors of $\mathbb{C}$. The action decomposes into irreps as follows: $\mathbb{C}^{4}=$ triv $\oplus$ triv $\oplus \omega \oplus \bar{\omega}$. Where $\omega$ is the 1 -dimensional representation given by $\rho((123))=\omega=\exp (2 \pi i / 3)$. The two trivial representations are spanned by $v_{1}=(1,0,0,0)$ and $v_{2}=(0,1,1,1)$ while the representation $\omega$ is spanned by $w=(0,1, \omega, \bar{\omega})$ and $\bar{\omega}$ by $\bar{w}$.

Although we cannot extract information about the degeneracies from this it helps greatly in determining the Eigenvalues, since there are two irreps with multiplicity one each giving a unique 1-dimensional Eigenspace for the Hamiltonian. Hence we immediately get two Eigenvalues. Plugging $w$ and $\bar{w}$ into $H(t)$ one reads off

$$
\lambda_{1}=\omega \exp (i a)+\bar{\omega} \exp (-i a) \quad \lambda_{2}=\bar{\omega} \exp (i a)+\omega \exp (i a)
$$




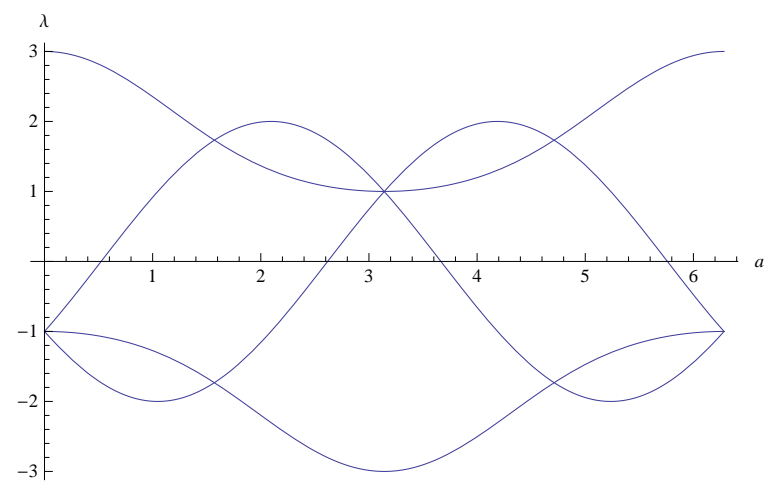

Figure 4. Spectrum of $H$ along the diagonal in $T^{3}$

The sum of the two trivial representations gives a 2-dimensional isotypical component. Therefore, we have to diagonalize $H$ inside this Eigenspace. It is interesting to note that at the special points it is exactly this flexibility that is needed in order to allow for crossings.

To determine the two remaining eigenvalues $\lambda_{3}$ and $\lambda_{4}$, we apply $H$ to $\vec{v}=x v_{1}+y v_{2}=(x, y, y, y)$. The eigenvalue equation $H \vec{v}=\lambda \vec{v}$ leads to the equations $3 y=\lambda x$ and $x+y(\exp (i a)+\exp (-i a))=\lambda y$, Fixing $x=3$ this gives the quadratic equation $\lambda^{2}-2 \cos (a) \lambda-3=0$ which has the two solutions

$$
\lambda_{3,4}=\cos (a) \pm \sqrt{\cos ^{2}(a)+3}
$$

This gives the spectrum along the diagonal which is given in Figure 4. The calculation only involves the classical symmetries without regauging.

In loc. cit. the authors also assert that numerically they only found singular points in the spectrum along the diagonal. The fact that the arising candidates for Dirac points are indeed such points and the analytic proof that indeed there are no other singular points in the spectrum is contained in [3].

4.2. The $\mathbf{P}$ case. There is nothing much to say here. There is only the root of the spanning tree which is unique. The $\mathbb{S}_{3}$ action permutes the edges and their weights. This yields the permutation action on the $T^{3}$. There is no nontrivial cover and the Eigenvalues remain invariant.

4.3. The $\mathbf{D}$ case. Here things again become interesting. Permuting the two vertices, we obtain eight fixed points if $a, b, c \in\{1,-1\}$. The matrix for this transposition is $\left(\begin{array}{ll}0 & 1 \\ 1 & 0\end{array}\right)$. This gives super-selection rules and we know that $v_{1}=(1,1)$ and $v_{2}=(-1,1)$ are Eigenvectors. 
The Eigenvalues being $1+a+b+c$ and $-(1+a+b+c)$ at these eight points.

We can also permute the edges with the $\mathbb{S}_{4}$ action. In this case the $\mathbb{S}_{3}$ action leaving the spanning tree edge invariant acts as a permutation on $(a, b, c)$. The relevant matrices however are just the identity matrices and the representation is trivial. The transposition (12), however, results in the action $(a, b, c) \mapsto(\bar{a}, \bar{a} b, \bar{a} c)$ on $T^{3}$, see Figure 3 . So to be invariant we have $a=1$, but this implies that $\rho_{12}$ is the identity matrix. Invariance for (13) and (14) and the three cycles containing 1 are similar. But, if we look at invariance under the element (12)(34) we are lead to the equations

$$
a=\bar{a}, b=\bar{a} c, c=\bar{a} b
$$

This has solutions $a=1, b=c$, for these fixed points again we find only a trivial action. But for $a=-1, b=-c$ these give rise to the diagonal matrix $\operatorname{diag}(1,-1)$ and hence Eigenvectors $e_{1}=(1,0)$ and $e_{2}=(0,1)$, but looking at the Hamiltonian, these are only Eigenvectors if it is the zero matrix $H(a, b, c)=0$. Indeed the conditions above imply $1+a+b+c=0$.

Similarly, we find a $\mathbb{Z} / 2 \mathbb{Z}$ group for (13)(24) and (14)(23) yielding the symmetric equations $b=-1, a=-c$ and $c=-1, a=-b$. These are exactly the three circles found in [6]. Going to bigger subgroups we only get something interesting if the stabilizer group $G_{t}$ contains precisely two of the double transposition above. That is the Klein four group $\mathbb{Z} / 2 \mathbb{Z} \times \mathbb{Z} / 2 \mathbb{Z}$. The invariants are precisely the intersection points of the three circles given by $a=b=-1$ and $c=1$ and its cyclic permutations.

These are three lines of double degenerate Eigenvalue 0. These are not Dirac points since there is one free parameter and hence the fibers of the characteristic map of [3] are one dimensional which implies that the singular point is not isolated.

4.4. The honeycomb case. This is very similar to the D story. The vertex interchange renders the fixed points $a= \pm 1, b= \pm 1$ which have Eigenvectors $v_{1}, v_{2}$ as above and Eigenvalues $1+a+b$ and $-(1+a+b)$ respectively. The irreps of the $C_{3}$ action are triv $\oplus \omega$.

As far as the edge permutations are concerned the interesting one is the cyclic permutation (123) which yields the equations

$$
a=\bar{b}, b=\bar{b} a
$$

for fixed points. Hence $a^{3}=1$. We get non-trivial matrices at the points $(\omega, \bar{\omega})$ and $(\bar{\omega}, \omega)$. At these points $e_{1}, e_{2}$ are Eigenvectors with 
Eigenvalue 0 and $H=0$, since $1+a+b=1+\omega+\bar{\omega}=0$. They are exactly the Dirac points of graphene.

\section{Conclusion}

By considering re-gaugings, we have found the symmetry groups fixing the degeneracies of the PDG and honeycomb families of graph Hamiltonians. The symmetries we used were those induced by the automorphisms of the underlying graphs. In our specific examples, all the graphs were highly symmetric, and hence had large automorphism group. Here we stress that our symmetries are extended symmetries and not just the classical ones. The most instructive and interesting case is the action of the binary tetrahedral group giving rise to the Dirac points in the Gyroid network. Note that as dimension-0 objects, the Dirac points for the Gyroid are codimension-3 defects in $T^{3}$, rather than codimension- 2 defects in $T^{2}$, the case for the honeycomb lattice, which describes graphene. Nevertheless, one may expect that they too lead to special physical properties.

There are several questions and research directions that tie into the present analysis.

It would be interesting to find concrete examples of lifts of regaugings either in the non-commutative case or in the case of regaugings not induced by graph symmetries. One place where we intend to look for the former is in the non-commutative case of PDG and the honeycomb as we aim to probe the non-commutative/commutative symmetry mentioned in Remark $§ 3.7$.

We are furthermore interested in how these symmetries behave under deformations of the Hamiltonian and if they are topologically stable. A physically important type of deformations are those corresponding to periodic (in space) lattice distortions that describe crystals with lower spatial symmetry than those considered here. Such distortions may occur for instance during synthesis of the structure [1]. Codimension-3 Dirac points, such as those of the Gyroid network, are especially interesting in this respect: they can be viewed as magnetic monopoles in the parameter space [13] and as such are topologically stable. This makes the physics associated with such points immune to periodic lattice distortions.

Finally it seems that on the horizon there are connections between our theory and two other worlds. The first being quiver representations in general and the second being cluster algebras. The connection to the first is inherent in the subject matter, while the connection to the second needs some work. The point is that in our transformations, 
we change several variables at a time. Nevertheless, the re-gauging groupoid can be viewed as a sort of mutation diagram. We plan to investigate these intriguing connections in the future.

\section{ACKNOWLEDGMENTS}

RK thankfully acknowledges support from NSF DMS-0805881. BK thankfully acknowledges support from the NSF under the grant PHY0969689. Any opinions, findings and conclusions or recommendations expressed in this material are those of the authors and do not necessarily reflect the views of the National Science Foundation.

Parts of this work were completed when RK was visiting, the IHES in Bures-sur-Yvette, the Max-Planck-Institute in Bonn and the University of Hamburg with a Humboldt fellowship. He gratefully acknowledges their support.

We also wish to thank Sergey Fomin for a short but very valuable discussion.

\section{REFERENCES}

[1] V.N. Urade, T.C. Wei, M.P. Tate and H.W. Hillhouse. Nanofabrication of double-Gyroid thin films. Chem. Mat. 19, 4 (2007) 768-777

[2] R.M. Kaufmann, S. Khlebnikov, and B. Wehefritz-Kaufmann, The geometry of the double gyroid wire network: quantum and classical, preprint arXiv:1010.1709, accepted for publication in Journal of Noncommutative Geometry

[3] R.M. Kaufmann, S. Khlebnikov, and B. Wehefritz-Kaufmann, Methods for graph Hamiltonians and applications to the Gyroid wire network I: Singularities, swallowtails and Dirac points, DESY preprint 12-132, accepted for publication in Annals of Physics

[4] A. H. Castro Neto, F. Guinea, N. M. R. Peres, K. S. Novoselov, and A. K. Geim, The electronic properties of graphene, Rev. Mod. Phys. 81, 109 (2009)

[5] J. E. Avron, A. Raveh and B. Zur, Adiabatic quantum transport in multiply connected systems, Rev. Mod. Phys. 60, 873 (1988)

[6] R.M. Kaufmann, S. Khlebnikov, and B. Wehefritz-Kaufmann, The noncommutative geometry of wire networks from triply periodic surfaces, J. Phys.: Conf. Ser. 343 (2012), 012054

[7] J. Bellissard, Gap labelling theorems for Schrödinger operators, in: From number theory to physics (1992) 538-630

[8] J.-P. Serre. Cohomologie galoisienne. Fifth edition. Lecture Notes in Mathematics, 5. Springer-Verlag, Berlin, 1994

[9] I. Schur. Über die Darstellung der symmetrischen und der alternierenden Gruppe durch gebrochene lineare Substitutionen. J. für Math. 139, 155-250 (1911).

[10] G. Karpilovsky, Projective Representations of Finite Groups, Dekker, 1985.

[11] K. S. Brown. Cohomology of Groups, Graduate Texts in Mathematics, 87, Springer Verlag, Berlin 1972. 
34 RALPH M. KAUFMANN, SERGEI KHLEBNIKOV, AND BIRGIT KAUFMANN

[12] I. Schur. Untersuchungen über die Darstellung der endlichen Gruppen durch gebrochene lineare Substitutionen. J. für Math. 132, 85-137 (1907)

[13] M. V. Berry, Quantum phase factors accompanying adiabatic changes, Proc. R. Soc. Lond. A 392, 45-57 (1984)

E-mail address: rkaufman@math.purdue.edu

Department of Mathematics, Purdue University, West Lafayette, IN 47907

E-mail address: skhleb@physics.purdue.edu

Department of Physics, Purdue University, West Lafayette, IN 47907

E-mail address: ebkaufma@math.purdue.edu

Department of Mathematics and Department of Physics, Purdue UNIVERSITY 